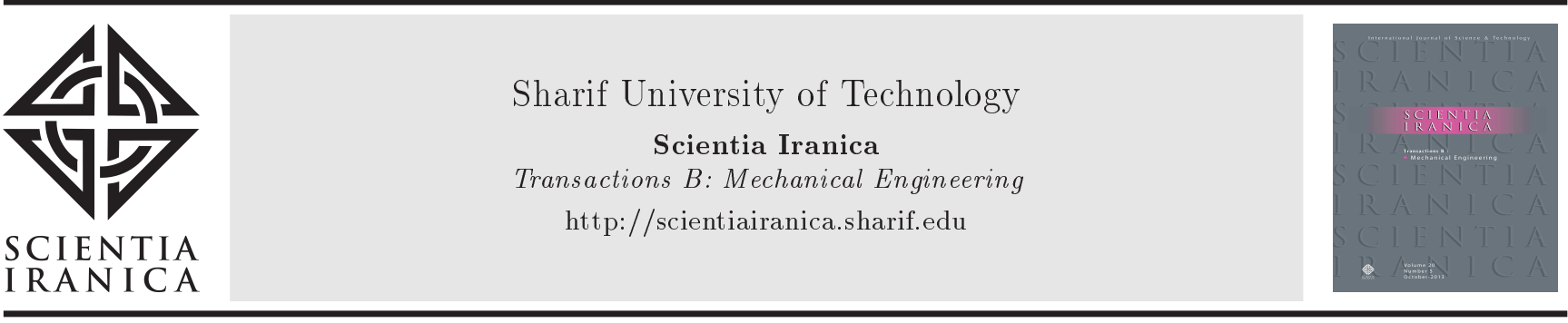

\title{
Design of a model reference controller for a dual-mode power-split hybrid electric vehicle during mode shift
}

\author{
C. Xiang and K. Huang* \\ National Key Laboratory of Vehicle Transmission, Beijing Institute of Technology, Beijing 100081, China.
}

Received 17 June 2016; received in revised form 18 October 2016; accepted 28 January 2017

\author{
KEYWORDS \\ Dual-mode power- \\ split Hybrid Electric \\ Vehicle (HEV); \\ Mode shift; \\ Switched system \\ theory; \\ Model reference \\ controller; \\ Shift quality.
}

\begin{abstract}
This paper presents a Model Reference Controller (MRC) of mode shift that intends to decrease the vehicle jerk and the clutch frictional loss for a dual-mode powersplit Hybrid Electric Vehicle (HEV). To design a model-based control system in this paper, simplified dynamic equations capturing mode shift dynamics of the dual-mode power-split HEV are derived. To simplify the complicated dynamic characteristics of mode shift, switched system theory is applied to partition the state space of mode shift into domains and facilitate the controller design. To deal with the friction-induced discontinuity of the clutch torque during mode shift, an MRC is proposed that coordinately manages the engine torque, the motor-generator torque, and the clutch friction torque. In addition, because the control system is overactuated by three control variables (three torques) and two output variables (two angular speeds), the controller parameter selections that involve selecting the combination of the control variables and the feedback-feedforward parameters are comparatively analyzed. The simulation and the experimental results demonstrate that the proposed MRC in this paper can simultaneously reduce the vehicle jerk and the clutch frictional loss, thereby improving the shift quality, when compared with the conventional controller.

(C) 2018 Sharif University of Technology. All rights reserved.
\end{abstract}

\section{Introduction}

Hybrid Electric Vehicles (HEVs) can be classified into three categories: series, parallel, and power-split HEVs [1]. The power-split HEVs, which consist of one engine and one or more Motor-Generators (MGs) combined with Planetary Gears (PGs), have attracted much attention from the automotive industry, because the power-split HEVs can be designed to take advantage of both series and parallel architectures, while simultaneously avoiding the disadvantages associated with each. When operating a power-split HEV, the PGs serve as a Power-Split Transmission (PST) that

*. Corresponding author. Tel.: +86 18810778622 E-mail address: bitkunhuang@gmail.com (K. Huang)

doi: $10.24200 /$ sci. 2017.4501 transfers the engine power to the vehicle through the mechanical path and the electrical path, as shown in Figure 1. The mechanical path transmits the power through the PST, which is directly coupled to the wheels. The rest of the engine power is transferred by the electrical path and is then transformed into electricity through the generator; next, this power is either sent to the motor or stored in the battery $[2,3]$. Because of the coordinated operation among the engine, the PST, and the MGs, the power-split $\mathrm{HEV}$ can provide a continuously variable transmission function and allow regulation of the engine speed in a vehicle speed-independent manner. Moreover, the MGs can coordinate engine starting and stopping while the vehicle is running. Therefore, these characteristics can deliver optimal engine operation to achieve better engine efficiency and lower emissions [4].

To enable the power-split HEVs at high efficien- 


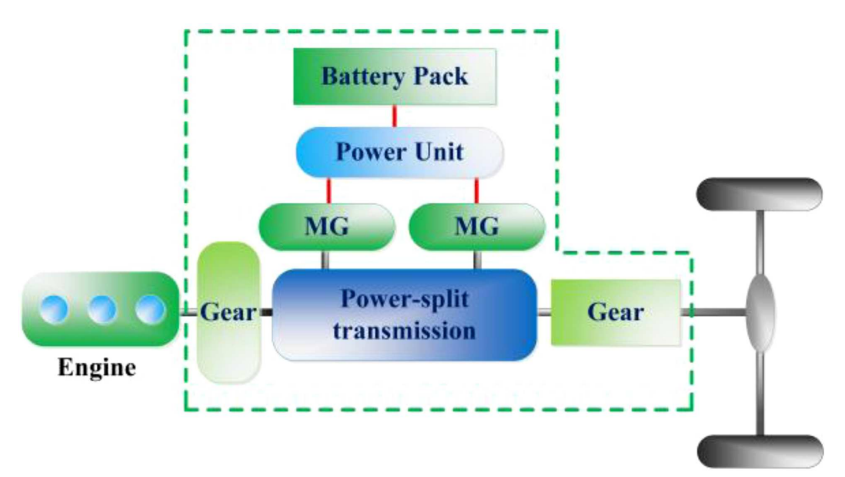

Figure 1. Configuration of power-split hybrid electric vehicle.

cies over a wide variety of operating conditions, dualmode power-split HEV has been introduced since the PST combines with a relatively small number of frictional elements, including clutch and brake, which incorporates supplemental power receiving-delivery units (engine, MGs, battery pack, etc.), such that the transmission is capable of providing enhanced tractive effort over wide speed range and large transmission power to meet the special requirements of heavy-load vehicles; therefore, the dual-mode power-split HEV is readily adaptable to the agricultural market or the commercial market. Previous research publications have focused on the dual-mode power-split HEVs because of the great potential to reduce carbon emission and improve fuel economies. A mode control strategy that considers the driving torque demand was implemented to minimize the fuel consumption of the dual-mode powersplit hybrids [5]. A rule-based supervisory control strategy was proposed to optimize fuel consumption by incorporating dynamic programming [6]. An optimal control strategy was applied to a new multiplemode power-split HEV to realize good fuel economy and emission performance [7]. Economical launching equipped with accelerating control strategy [8] and a corrected stochastic model predictive control [9] were proposed to improve the fuel economy of a multiplemode power-split HEV. In addition, to improve the overall powertrain efficiency, inversion-based control applied to design motor torque control algorithm [10], optimal operation line control to manage the engine operation point [11], and fuzzy logical control to sustain the battery state of charge [12] were proposed for the dual-mode power-split hybrids.

To make full use of the dual-mode system topology, mode shift as a significant phase among the different operating modes is necessary to maintain the vehicle stability. However, an inappropriate mode shift, if improperly managed, might reduce shift quality of the vehicle. This poor shift quality is caused by the occurrence of torque interruption when the clutch (brake) is improperly engaged during mode shift. In this scenario, handling the friction-induced discontinuity of the clutch (brake) torque to achieve a smooth mode shift is the main challenge in future development of the dual-mode power-split HEV.

The clutch (brake) is a key operational component of the dual-mode power-split $\mathrm{HEV}$ to connect the powertrain system or transfer the power sources to manage the vehicle running. Basically, the process of clutch engagement can be divided into three stages, namely, open process, slipping process, and locked process. For the slipping process, the angular speeds of the driving-driven shaft are desynchronized, and thus two degrees of freedoms exist in the clutch model. Regarding the clutch-coupled transmission as the control structure of dual-mode power-split HEV, normally, the control system comprising two degrees of freedom is manipulated by three control variables, namely, engine torque, clutch friction torque, and $\mathrm{MG}$ torque, that obviously make the control system an overactuated system during the slipping process. To achieve the ideal synchronization condition of the clutch, a systematic approach is developed for this overactuated system in this paper that intends to select a suitable combination of control variables and formulate the criterion to manage these parameters.

Besides, the slipping-locked transition process can easily induce the discontinuity of the clutch torque, and the sharp change of the frictional torque may lead to intensive vehicle jerk. Therefore, to reduce the vehicle jerk, various research studies on the transient state due to the slipping-locked transition process of the clutch have investigated optimal control design of the clutch for a vehicle equipped with a dual-clutch transmission [13], model predictive control method applied to regulate the clutch torque $[14,15]$, slipping-speed control approach of the clutch [16], and the robust controller proposed for the clutchto-clutch shift [17]. In addition, the conventional control strategies that define a predefined friction torque profile of the clutch associated with engine torque modulation [18] and MG torque control [19] can also smooth the slipping-locked transition process and shift quality improvement. Existing solutions found in the above-described research publications are based on heuristic techniques; however, these control system designs do not explicitly exploit the modelling aspect involving the clutch model. Therefore, selecting a model-based methodology [20] to present a systematic design architecture is one of the primary interests in this paper, and may have the possibility to achieve the expected control goals.

The objective of this paper is to propose a Model Reference Controller (MRC) that is used for dualmode power-split HEV to achieve smooth mode shift and improved shift quality with reduced vehicle jerk and less clutch frictional loss. The objectives can be achieved by the following: 
1. The detailed configuration of the dual-mode powersplit $\mathrm{HEV}$ is first presented, and the controloriented model to capture the mode shift dynamics is derived;

2. Based on switched system theory, the dual-mode power-split HEV is considered as a switched system model, and the mode shift is partitioned into three domains to facilitate the controller design;

3. Under the architecture of the MRC, a reference model is developed to describe the expected control target of mode shift, a systematic method is applied to choose a proper combination of the control variables, a feedforward controller is designed to achieve the constraint equations of the reference variables, and a feedback controller is proposed to determine the feedback parameters based on the stability conditions of the control system;

4. The simulation results and the experimental results demonstrate the effectiveness of the proposed MRC, when compared with a control system equipped with a conventional controller.

\section{Dual-mode power-split HEV}

\subsection{Introduction to the dual-mode power-split $\boldsymbol{H} \boldsymbol{E} \boldsymbol{V}$}

Figure 2 depicts the overall layout of the dual-mode power-split HEV, which mainly consists of three Planetary Gears (PGs), two Motors/Generators (MGs), one clutch, one brake, and battery. The three PGs are used as a PST that provides the interconnection between the engine and MGs, and each planetary gear consists of a sun gear, a ring gear, and a carrier gear that carries a set of small gears called pinion gears. It is seen that the engine engages with the carrier gear of PG1 and that MG1 is connected to the ring gear of PG2. The sun gear and the ring gear of PG1 are connected to the sun gear and the carrier of PG2, respectively. In

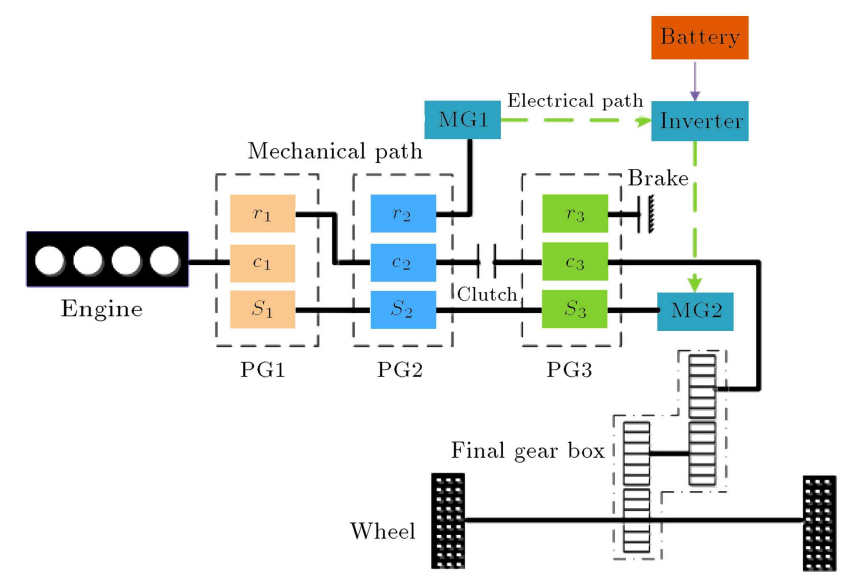

Figure 2. Configuration of the dual-mode power-split HEV.
Table 1. Operation modes of the dual-mode power-split HEV.

\begin{tabular}{ccccc}
\hline Mode & MG1 & MG2 & Clutch & Brake \\
\hline EVT1 & Generator & Motor & $\times$ & $\circ$ \\
EVT2 & Motor & Generator & $\circ$ & $\times$ \\
\hline
\end{tabular}

$\mathrm{x}$ : Disengagement; o: Engagement.

addition, MG2 is connected to the sun gear of PG3 and the output shaft is connected to the carrier of PG3.

The dual-mode power-split HEV can provide two Electrically Variable Transmission (EVT) modes by operating the MGs and the clutch (brake), as presented in Table 1. When the clutch is disengaged (and the brake is engaged), the vehicle operates in the EVT1 mode. In this case, MG1 is used as a generator and MG2 behaves as a motor, that is, MG2 generates positive torque to propel the vehicle running, which can positively regulate the engine operation to the optimal operation line, and the electrical power generated by MG1 is transmitted to MG2 or stored in the battery. On the other hand, when the clutch is engaged (and the brake is disengaged), MG1 is used as a motor and MG2 behaves as a generator. This case defines the EVT2 mode; this means that MG1 is applied to maintain the engine optimal operation points, and the electrical power from MG2 is supplied to MG1 and the battery. Therefore, the mode shift from EVT 1 to EVT 2 is a critical and necessary process to transfer power sources among the engine, MGs, and battery pack.

\subsection{Problem formulation}

Simulation and experimental results [21-23] focused on the dual-mode power-split HEVs with similar configurations have shown that the variation in the transient torque due to the engagement of the clutch during mode shift can cause intensive vehicle jerk and reduced shift quality. In this paper, the mode shift of the dual-mode power-split HEV from EVT 1 to EVT 2 can be implemented by three steps due to the process of clutch engagement. Firstly, the clutch is in the open process and the driving-driven shafts are not in contact; therefore, no clutch friction torque exists. The vehicle is manipulated by energy management strategy to coordinate the torques of the engine and MGs. Moreover, due to the engine speed regulation, the angular speed difference of driving-driven shafts can be regulated to a specific value. Secondly, as the engine torque and MGs torque change sharply, the clutch enters the slipping process. The clutch friction torque increases gradually, which is controlled by the oil pressure actuators, while that in the third step is not because the clutch is fully engaged during the clutch-locked process. In other words, the clutch friction torque during the slipping process may become discontinuous and change suddenly to cause intensive vehicle jerk. Therefore, a systematic design method 
on how to manage the torque coordination during the clutch-slipping phase, i.e., coordinating en gine torque, clutch friction torque, and MG torque, is the main challenge of mode shift and is also the central topic of this paper.

\subsection{Dynamic model of the dual-mode power-split $\mathrm{HEV}$}

To explicitly investigate the dynamics of mode shift and design a model-based control system in this paper, the configuration of the dual-mode power-split HEV is simplified into a clutch-coupled transmission topology, as shown in Figure 3. To simplify the modeling, here, the mass of each planetary gear is assumed to be concentrated at its axis of rotation and all the driveline shafts are considered as rigid bodies, making the vehicle powertrain system involving the clutch engagement a lumped-mass system. Therefore, the total moment of inertia, involving the engine, MG1, PG1, and PG2, is concentrated on shaft 1 , and the other total moment of inertia, involving MG2, PG3, and vehicle body, is concentrated on shaft 2. Besides, the drivingshaft of the clutch is connected to shaft 1 and the driven-shaft is connected to shaft 2 , as shown in Figure 3(b).

The torque equations and speed equations of the dual-mode power-split HEV can be obtained by using a lever analogy, and the detailed derivation process can be found in $[5,19]$. Thus, the torque relationships between Figure 3(a) and (b) can be expressed as follows:

$$
\begin{aligned}
& T_{\mathrm{MG} 1}(t)=\frac{K_{1}}{1+K_{1}} T_{1}(t) \\
& T_{\mathrm{MG} 2}(t)=\frac{1+K_{1}+K_{2}}{K_{2}\left(1+K_{1}\right)} T_{1}(t)+\frac{1}{1+K_{3}} T_{2}(t),
\end{aligned}
$$

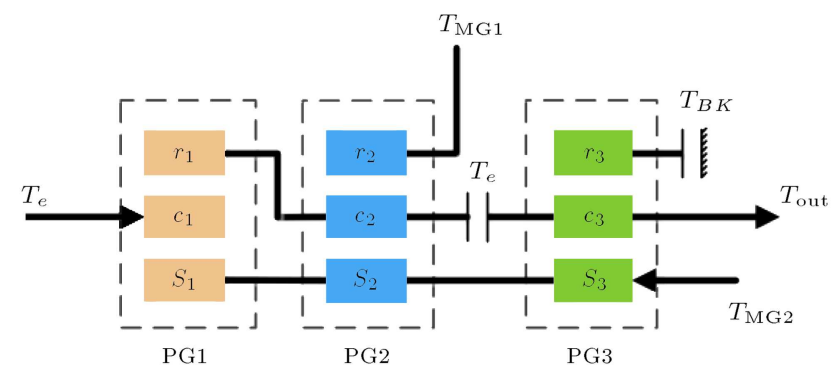

(a)

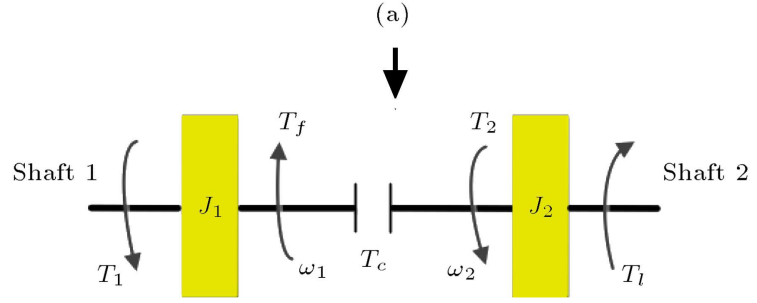

(b)

Figure 3. Dynamic model of the dual-mode power-split HEV: (a) Driveline model, and (b) simplified model.

$$
\begin{aligned}
T_{e}(t)=- & \frac{\left(1+K_{1}\right)\left(1+K_{2}\right)}{K_{1} K_{2}} T_{\mathrm{MG} 1}(t), \\
T_{\text {out }}(t)= & -\frac{\left(1+K_{1}+K_{2}\right)\left(1+K_{3}\right)}{\left(1+K_{1}\right)\left(1+K_{2}\right)} T_{\mathrm{MG} 1}(t) \\
& +\left(1+K_{3}\right) T_{\mathrm{MG} 2}(t),
\end{aligned}
$$

where subscript 1 denotes PG1, subscript 2 denotes PG2, and subscript 3 denotes PG3; $T_{e}, T_{\text {out }}, T_{\mathrm{MG} 1}$, and $T_{\mathrm{MG} 2}$ are the engine torque, the output torque, MG1 torque, and MG2 torque, respectively; the engine torque, $T_{e}$, can be obtained by the engine throttle opening and the engine speed, the $\mathrm{MG}$ torque, $T_{\mathrm{MG}}$, can be modelled through a map of the MG's characteristic and a map of the efficiency [19]; $T_{1}$ and $T_{2}$ are the equivalent torques concentrated on shaft 1 and shaft 2 , respectively. It is seen that $T_{1}$ directly reflects the engine torque, $T_{e}$, and $M_{G 1}$ torque, $T_{\mathrm{MG} 1}$; and $T_{2}$ involves the engine torque, $T_{e}$, and $M_{G 2}$ torque $T_{\mathrm{MG} 2}$. Therefore, three control torques of the dualmode power-split HEV, involving the engine torque, MG1 torque, and MG2 torque, have been integrated into two equivalent torques $T_{1}$ and $T_{2}$, which can capture the dynamics of the engine torque and MGs torque. $K$ represents the characteristic parameter of the PGs, which can be written as:

$$
K_{j}=\frac{Z_{r j}}{Z_{s j}}, \quad j=1,2,3
$$

where $Z_{s}$ is the number of teeth on the sun gear and $Z_{r}$ is the number of teeth on the ring gear. To develop a control-oriented model for the control system design, the dynamic equations of the mode shift can be expressed as:

$$
\begin{aligned}
& J_{1} \dot{\omega}_{1}(t)=T_{1}(t)-T_{f}(t)-T_{c}(t), \\
& J_{2} \dot{\omega}_{2}(t)=T_{2}(t)+T_{c}(t)-T_{l}(t),
\end{aligned}
$$

where $J_{1}$ is the equivalent moment of inertia of shaft 1 including the engine, MG1, and the driving shaft of the clutch; $J_{2}$ is the equivalent moment of inertia of shaft 2 including the vehicle body, MG2, and the driven shaft of the clutch; $\omega_{1}$ is the angular speed of shaft 1 , which is proportional to the engine speed and the MG1 speed; $\omega_{2}$ is the angular speed of shaft 2 , which is proportional to the vehicle speed and the MG2 speed; $T_{c}$ is the clutch friction torque; $T_{f}$ is the resistance torque of shaft 1 produced by the engine-startup process; $T_{l}$ is the resistance torque of shaft 2 including the rolling resistance, the drag force, and the grade resistance [24]. The clutch model aspect is highlighted in this section, and the clutch friction torque due to the engagement process can be expressed as: 


$$
T_{c}= \begin{cases}0 & \text { (open) } \\ Z \mu_{d} \frac{2}{3} \frac{R_{o}^{3}-R_{i}^{3}}{R_{o}^{2}-R_{i}^{2}} P A_{p} \operatorname{sgn}\left(\omega_{1}-\omega_{2}\right) & \text { (slipping) } \\ Z \mu_{s} \frac{2}{3} \frac{R_{o}^{3}-R_{i}^{3}}{R_{o}^{2}-R_{i}^{2}} P A_{p} & \text { (locked) }\end{cases}
$$

where $Z$ is the number of clutch discs, $R_{o}$ is the outside radius of the clutch plate, $R_{i}$ is the inside radius of the clutch plate, $P$ is the hydraulic pressure of the clutch, $A_{p}$ is the clutch piston area, $\mu_{d}$ is the dynamic friction coefficient of clutch, and $\mu_{s}$ is the static friction coefficient of the clutch.

Furthermore, when $\omega_{1}=\omega_{2}$ is achieved in the locked process, the clutch friction torque can be given through the calculation of Eqs. (6) and (7) by:

$$
\begin{aligned}
T_{c}= & \frac{J_{2}}{J_{1}+J_{2}} T_{1}(t)-\frac{J_{2}}{J_{1}+J_{2}} T_{f}(t)-\frac{J_{1}}{J_{1}+J_{2}} T_{2}(t) \\
& +\frac{J_{1}}{J_{1}+J_{2}} T_{l}(t) .
\end{aligned}
$$

Therefore, the dynamic equations of the mode shift can be restructured as:

1. For the open process, $T_{c}(t)=0$ :

$$
\left\{\begin{array}{l}
J_{1} \dot{\omega}_{1}(t)=T_{1}(t)-T_{f}(t) \\
J_{2} \dot{\omega}_{2}(t)=T_{2}(t)-T_{l}(t)
\end{array}\right.
$$

2. For the slipping process, $\omega_{1} \neq \omega_{2}$ :

$$
\left\{\begin{array}{l}
J_{1} \dot{\omega}_{1}(t)=T_{1}(t)-T_{f}(t)-T_{c}(t) \\
J_{2} \dot{\omega}_{2}(t)=T_{2}(t)+T_{c}(t)-T_{l}(t) \\
T_{c}(t)=Z \mu_{d} \frac{2}{3} \frac{R_{o}^{3}-R_{i}^{3}}{R_{o}^{2}-R_{i}^{2}} P A_{p} \operatorname{sgn}\left(\omega_{1}-\omega_{2}\right)
\end{array}\right.
$$

3. For the locked process, $\omega_{1}=\omega_{2}=\omega_{m}$ :

$$
\left\{\begin{array}{c}
\left(J_{1}+J_{2}\right) \dot{\omega}_{m}(t)=T_{1}(t)+T_{2}(t)-T_{f}(t)-T_{l}(t) \\
T_{c}(t)=\frac{J_{2}}{J_{1}+J_{2}} T_{1}(t)-\frac{J_{2}}{J_{1}+J_{2}} T_{f}(t) \\
-\frac{J_{1}}{J_{1}+J_{2}} T_{2}(t)+\frac{J_{1}}{J_{1}+J_{2}} T_{l}(t) \\
T_{c}(t) \in\left[-Z \mu_{s} \frac{2}{3} \frac{R_{o}^{3}-R_{i}^{3}}{R_{o}^{2}-R_{i}^{2}} P A_{p}\right. \\
\left.Z \mu_{s} \frac{2}{3} \frac{R_{o}^{3}-R_{i}^{3}}{R_{o}^{2}-R_{i}^{2}} P A_{p}\right]
\end{array}\right.
$$

At this point, the control-oriented model of the dualmode power-split HEV capturing the mode shift dynamics has been explicitly developed and presented.

\section{Switched system theory application}

To simplify the complicated dynamic characteristics of mode shift, switched system theory has been applied to partition the operational modes of mode shift into different domains, which can provide a systematic methodology to facilitate the modelling and the controller design for each domain.

\subsection{Introduction to switched system}

Switched system is a class of dynamical systems that consists of continuous-time systems interrupted by discrete-time event. Typically, a class of switched system with multiple modes can be described as [2528]:

$$
\Sigma=\bigcup_{i=1}^{m} \Sigma_{i}
$$

where $\Sigma_{i}$ represents each mode of the switched system that can be denoted by the seven-tuple model:

$$
\Sigma_{i}:\left\{X_{i}, P_{i}, U_{i}, F_{i}, T_{i}^{j}, S_{i}^{j}, J_{i}^{j}\right\}
$$

where $X_{i} \subset \mathbb{R}^{n_{i}}$ is the associated state space, $P_{i}=$ $\left\{P_{i}^{0}, P_{i}^{1}, \cdots, P_{i}^{d_{i}}\right\}$ such that $\cup_{k=0}^{d_{i}} P_{i}^{k}=X_{i}$ are the $d_{i}+1$ partitions of $X_{i}, U_{i}=\left\{u_{i}^{0}, u_{i}^{1}, \cdots u_{i}^{d_{i}}\right\}$ is the $d_{i}+1$ control variables on the corresponding partitions where $u_{i}^{k}: \mathbb{R} \times X_{i} \rightarrow \mathbb{R}^{p_{i}}, F_{i}$ is the associated vector field on $X_{i}$ where $F_{i}: \mathbb{R} \times X_{i} \times U_{i} \rightarrow \mathbb{R}^{n_{i}}, T_{i}^{j}: X_{i} \rightarrow X_{j}$ is the switching rule from mode $i$ to mode $j, S_{i}^{j} \subset X_{i}$ is the sets of $T_{i}^{j}$, and $J_{i}^{j}: S_{i}^{j} \rightarrow \mathbb{R}_{+}$is the associated cost functions shifting from mode $i$ to mode $j$.

Basically, the controller design of the switched system consists of two steps. In the first step, $S$ should be chosen to make the cost function $\|J(S)\|$ minimized, where $\|\bullet\|$ is associated norm; in the second step, $X$ is partitioned to different modes and the associated controllers are designed for each mode.

\subsection{Switched system theory applied to dual-mode power-split $\mathrm{HEV}$}

The mode shift process of the dual-mode power-split $\mathrm{HEV}$ is the combination of different continuous-time systems, and each operational mode, i.e. EVT 1 or EVT 2, can be seen as a steady status. In addition, shifting from one status to another status needs the assistance of the associated discrete-time event. Therefore, the dual-mode power-split $\mathrm{HEV}$ involving the mode shift dynamics is a typical class of the switched system.

Eqs. (10)-(12) present the dynamics of the mode shift from EVT 1 to EVT 2, and an overall switched system model can be constructed using the abovedescribed modeling formalism. The state variables are chosen as $X_{1}:=\left(\omega_{1}, \omega_{2}\right)^{\prime}, X_{2}:=\left(\omega_{1}, \omega_{2}\right)^{\prime}$, and $X_{3}:=\left(\omega_{m}\right)$. The partitions of $X_{i}$ are given by $P_{1}:=$ $\left\{P_{1}^{0}, P_{1}^{1}\right\}, P_{1}^{0}:=\left(\omega_{1}\right)^{\prime}, P_{1}^{1}:=\left(\omega_{2}\right)^{\prime} ; P_{2}:=\left\{P_{2}^{0}, P_{2}^{1}\right\}$, 
$P_{2}^{0}:=\left(\omega_{1}\right), P_{2}^{1}:=\left(\omega_{2}\right) ;$ and $P_{3}:=\left\{P_{3}^{0}\right\}, P_{3}^{0}:=\left(\omega_{m}\right)$. The control variables are expressed by $U_{1}:=\left\{u_{1}^{0}, u_{1}^{1}\right\}$, $u_{1}^{0}:=\left(T_{1}\right), u_{1}^{1}:=\left(T_{2}\right) ; U_{2}:=\left\{u_{2}^{0}, u_{2}^{1}\right\}, u_{2}^{0}:=\left(T_{1}, T_{c}\right)^{\prime}$, $u_{2}^{1}:=\left(T_{2}, T_{c}\right)^{\prime}$; and $U_{3}:=\left\{u_{3}^{0}\right\}, u_{3}^{0}:=\left(T_{1}, T_{2}\right)^{\prime}$. The dynamics of the mode shift can be rewritten by the vector fields $F_{1}, F_{2}$, and $F_{3}$ as follows:

$$
\begin{aligned}
& F_{1}:=\left\{\begin{array}{l}
\dot{\omega}_{1}(t)=\frac{T_{1}(t)-T_{f}(t)}{J_{1}} \\
\dot{\omega}_{2}(t)=\frac{T_{2}(t)-T_{l}(t)}{J_{2}}
\end{array} \quad\right. \text { (open process) } \\
& F_{2}:=\left\{\begin{array}{lr}
\dot{\omega}_{1}(t)=\frac{T_{1}(t)-T_{f}(t)-T_{c}(t)}{J_{1}} & \\
\dot{\omega}_{2}(t)=\frac{T_{2}(t)+T_{c}(t)-T_{l}(t)}{J_{2}} & (\text { slipping process) }
\end{array}\right. \\
& F_{3}:=\dot{\omega}_{m}(t)=\frac{T_{1}(t)+T_{2}(t)-T_{f}(t)-T_{l}(t)}{\left(J_{1}+J_{2}\right)},
\end{aligned}
$$

(locked process)

In addition, the switching rules are proposed by $T_{1}^{2}$ : $X \rightarrow X$ and $T_{2}^{3}: X \rightarrow A X$, where $A=[01]^{\prime}$; the sets of the switching rule are $S_{1}^{2}:=\left\{\left|\Delta \omega_{c}\right| \leq \Delta \varpi_{c}\right\}$ and $S_{2}^{3}:=\left\{\left|\Delta \omega_{c}\right|=0\right\}$, where $\Delta \omega_{c}=\omega_{1}-\omega_{2}$ is the angular speed difference of the driving-driven shafts of the clutch and $\Delta \varpi_{c}$ is a specific value. The cost functions associated with the switching rules are $J_{1}^{2}:=\left|\Delta \omega_{c}\right|$ and $J_{2}^{3}:=\left|e_{1}\right|+\left|e_{2}\right|$, where $e_{1}$ and $e_{2}$ are the angular speed errors with $e_{1}=\omega_{1}-\omega_{m}$ and $e_{2}=\omega_{2}-\omega_{m}$. Minimizing the cost function $J_{1}^{2}$ is to ensure that the angular speed difference is approaching synchronization with $\Delta \varpi_{c}$, and minimizing the cost function $J_{2}^{3}$ is to ensure that the clutch speed is fully synchronized. Note that after the vehicle enters the clutch-locked process, the torque interruption cannot occur to impact on the vehicle's shift quality, so the switching rule, $T_{3}^{j}$, the switching set, $S_{3}^{j}$, and the cost function, $J_{3}^{j}$, are not specified. As a whole, the overall framework of the switched system for the dual-mode power-split HEV has been designed and the switched system model diagram of mode shift is shown in Figure 4.

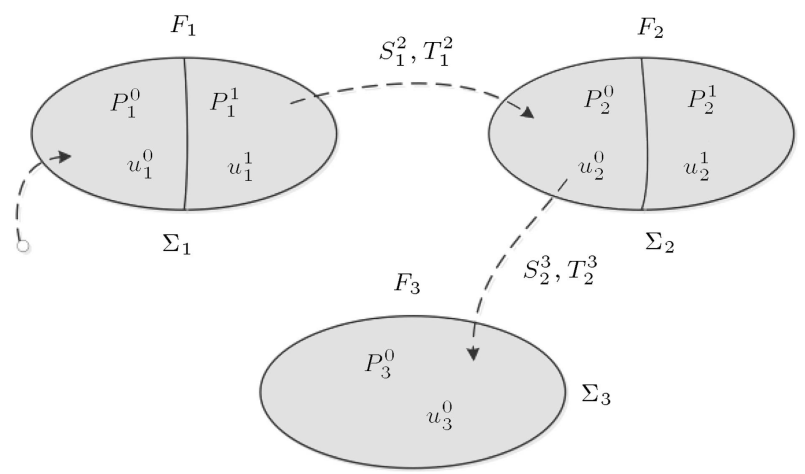

Figure 4. Switched system model diagram of mode shift.
At this point, the mode shift process of the dualmode power-split HEV has been simplified into three domains that are integrated into a control system, and this systematic methodology facilitates the controller design.

\section{Design for model reference controller}

For the clutch-open process, to minimize the cost function, $J_{1}^{2}$, with a specific angular speed difference, $\Delta \varpi_{c}$, usually, the controller can be designed by the energy management strategy that involves the management of the torque distribution and the power distribution among the engine, MGs, and battery. However, as described in Section 2.2, the controller design of the clutch-open process is not the focus of this paper. On the other hand, the clutch friction torque produced during the clutch-slipping process might become discontinuous and suddenly change, which can lead to intensive vehicle jerk. Therefore, a Model Reference Controller (MRC) design for the clutch-slipping process is of primary interest in this section, and the detailed design process is given in the following.

\subsection{Overall description of control system diagram}

Figure 5 presents the design diagram of the MRC of mode shift for the dual-mode power-split HEV. It is seen that a reference model, a control plant, a feedforward controller, and a feedback controller are included. The reference model generates a reference angular speed of the clutch that aims to constrain the dynamics of mode shift. The control plant captures the dynamic equation (11) during the clutch-slipping process to produce the actual angular speeds of the clutch, and $e(t)$ denotes the angular speed errors of the clutch including $e_{1}(t)$ and $e_{2}(t)$. In addition, the desired traction torque is introduced as a reference input variable, the feedforward controller generates reference input variables, and the feedback controller regulates feedback parameters to the control system.

To define the expected control target of the dualmode power-split HEV during mode shift, a reference model is introduced to ensure a seamless transition. In the reference model, a desired traction torque, $T_{m}$, concentrated on shaft 2 maintains the vehicle running,

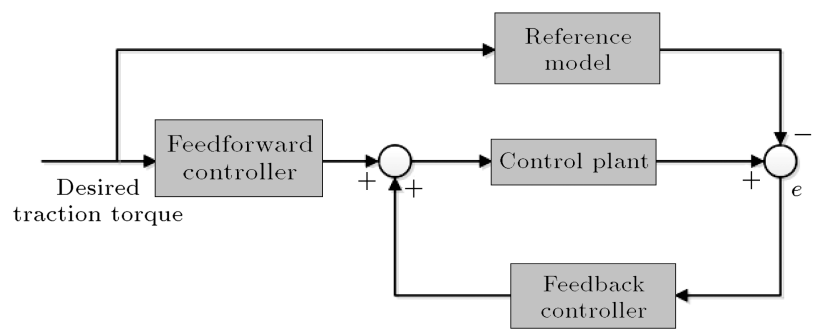

Figure 5. Model reference controller design diagram. 
and the clutch is supposed to be fully engaged so that the driving-driven shafts are synchronized with an identical angular speed, $\omega_{m}$. Consequently, the dynamic equation of the reference model can be expressed as:

$$
\left(J_{1}+J_{2}\right) \dot{\omega}_{m}(t)=T_{m}(t)-T_{f}(t)-T_{l}(t) .
$$

Besides, to facilitate the control system design, the control notations are defined by the following state variables:

$$
\begin{array}{ll}
\omega_{1}(t)=x_{1}(t), & \omega_{2}(t)=x_{2}(t), \\
y_{1}(t)=x_{1}(t), & y_{2}(t)=x_{2}(t), \\
u_{1}(t)=T_{1}(t), & u_{2}(t)=T_{2}(t), \quad u_{3}(t)=T_{c}(t) .
\end{array}
$$

$T_{f}$ and $T_{l}$ are assumed as the external disturbances that can be denoted by $d_{1}(t)=T_{f}(t)$, and $d_{2}(t)=$ $T_{l}(t)$. The state variable, $\omega_{m}(t)=x_{m}(t)$, the control variable, $u_{m}(t)=T_{m}(t)$, and the output variable, $y_{m}(t)=x_{m}(t)$, are used for the reference model. Furthermore, $r(t)$ is the desired traction torque with $r(t)=u_{m}(t)=T_{m}(t)$; and $r_{1}(t), r_{2}(t)$, and $r_{3}(t)$ denote the reference input variables of the feedforward controller. Consequently, the control goal is to achieve $J_{2}^{3}:=\left\{\mathbf{e}(t)|| e_{1}|+| e_{2} \mid=0\right\}$, and the systematic architecture of the control system based on the modelbased methodology can be developed by the following expressions and expressed by Figure 6:

1. Control plant model:

$$
\left\{\begin{array}{l}
J_{1} \dot{x}_{1}(t)=u_{1}(t)-u_{3}(t)-d_{1}(t) \\
J_{2} \dot{x}_{2}(t)=u_{2}(t)+u_{3}(t)-d_{2}(t) \\
y_{1}(t)=x_{1}(t) \\
y_{2}(t)=x_{2}(t)
\end{array}\right.
$$

2. Reference model:

$$
\left\{\begin{array}{l}
\left(J_{1}+J_{2}\right) \dot{x}_{m}(t)=u_{m}(t)-d_{1}(t)-d_{2}(t) \\
y_{m}(t)=x_{m}(t)
\end{array}\right.
$$

3. Input to the reference model and the controller:

$$
r(t)=u_{m}(t)=T_{m}(t) .
$$

4. Error vector of output variables:

$$
\mathbf{e}(t)=\left(\begin{array}{l}
e_{1}(t) \\
e_{2}(t)
\end{array}\right)=\left(\begin{array}{l}
y_{1}(t)-y_{m}(t) \\
y_{2}(t)-y_{m}(t)
\end{array}\right) .
$$

5. Proposed feedback-feedforward control algorithms:

$$
\left\{\begin{array}{l}
u_{1}(t)=r_{1}(t)+k_{11} e_{1}(t)+k_{12} e_{2}(t) \\
u_{2}(t)=r_{2}(t)+k_{21} e_{1}(t)+k_{22} e_{2}(t) \\
u_{3}(t)=r_{3}(t)+k_{31} e_{1}(t)+k_{32} e_{2}(t)
\end{array}\right.
$$

\subsection{Stability conditions of control system}

The error equations among the output variables in the proposed control system can be derived by the calculation of Eqs. (19)-(22), which transform into a state-space expression by Eqs. (24) and (25) as shown in Box I.

The necessary and sufficient condition to guarantee asymptotical stability of the error equations is that every eigenvalue of matrix $\mathbf{A}$ is supposed to have a strictly negative real part. Therefore, based on Lyapunov stability criterion, the stability conditions to constrain the closed-loop system can be derived as:

$$
\begin{aligned}
& k_{11}+k_{22}-k_{31}+k_{32}<0 \\
& k_{31}\left(k_{12}+k_{22}\right)+k_{21}\left(k_{12}-k_{32}\right)-k_{11}\left(k_{22}+k_{32}\right)<0 .
\end{aligned}
$$

\subsection{The combination of control variables}

Based on Eq. (19), it is found that the control system is overactuated with three control variables $\left(u_{1}(t), u_{2}(t), u_{3}(t)\right)$ and two output variables $\left(y_{1}(t), y_{2}(t)\right)$. Therefore, a proper combination selection of the control variables is necessary for this overactuated system. According to Eqs. (1)-(4), it is found that the equivalent torque, $T_{2}(t)$, provides the main positive driving torque of the vehicle running because the engine and MG2 are the main power sources of the dual-mode power-split HEV during mode shift. Therefore, $u_{2}(t)$ should be considered as a predefined control variable, and then three combinations with one predefined control variable can be given as: $\left(u_{1}(t), u_{2}(t)\right),\left(u_{2}(t), u_{3}(t)\right)$, and $\left(u_{1}(t), u_{2}(t), u_{3}(t)\right)$.

Typically, since the control variable, $u_{1}(t)$, is directly associated with the engine torque, less use of

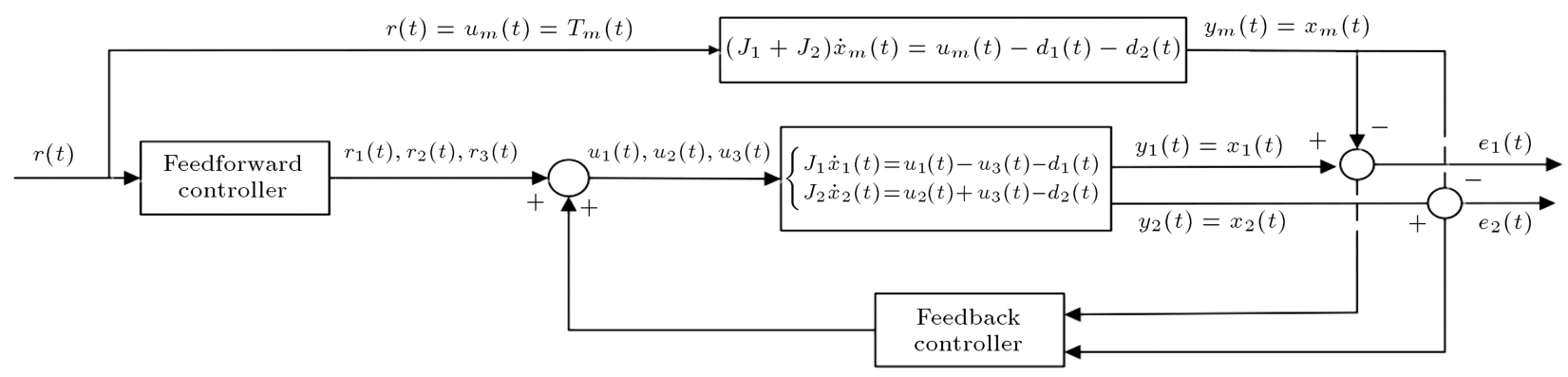

Figure 6. Systematic architecture of the control system. 


$$
\begin{aligned}
& \left(\begin{array}{l}
\dot{e}_{1}(t) \\
\dot{e}_{2}(t)
\end{array}\right)=\left(\begin{array}{l}
\dot{y}_{1}(t)-\dot{y}_{m}(t) \\
\dot{y}_{2}(t)-\dot{y}_{m}(t)
\end{array}\right)=\left(\begin{array}{c}
\frac{u_{1}(t)-u_{3}(t)-d_{1}(t)}{J_{1}}-\frac{u_{m}(t)-d_{1}(t)-d_{2}(t)}{J_{1}+J_{2}} \\
\frac{u_{2}(t)+u_{3}(t)-d_{2}(t)}{J_{2}}-\frac{u_{m}(t)-d_{1}(t)-d_{2}(t)}{J_{1}+J_{2}}
\end{array}\right) \\
& =\left(\begin{array}{l}
\frac{r_{1}(t)+k_{11} e_{1}(t)+k_{12} e_{2}(t)-r_{3}(t)-k_{31} e_{1}(t)-k_{32} e_{2}(t)-d_{1}(t)}{J_{1}}-\frac{r(t)-d_{1}(t)-d_{2}(t)}{J_{1}+J_{2}} \\
\frac{r_{2}(t)+k_{21} e_{1}(t)+k_{22} e_{2}(t)+r_{3}(t)+k_{31} e_{1}(t)+k_{32} e_{2}(t)-d_{2}(t)}{J_{2}}-\frac{r(t)-d_{1}(t)-d_{2}(t)}{J_{1}+J_{2}}
\end{array}\right) \\
& =\left(\begin{array}{cc}
\frac{k_{11}-k_{31}}{J_{1}} & \frac{k_{12}-k_{32}}{J_{1}} \\
\frac{k_{21}+k_{31}}{J_{2}} & \frac{k_{22}+k_{32}}{J_{2}}
\end{array}\right)\left(\begin{array}{l}
e_{1}(t) \\
e_{2}(t)
\end{array}\right)+\left(\begin{array}{cccc}
\frac{-1}{J_{1}+J_{2}} & \frac{1}{J_{1}} & 0 & \frac{-1}{J_{1}} \\
\frac{-1}{J_{1}+J_{2}} & 0 & \frac{1}{J_{2}} & \frac{1}{J_{2}}
\end{array}\right)\left(\begin{array}{c}
r(t) \\
r_{1}(t) \\
r_{2}(t) \\
r_{3}(t)
\end{array}\right)+\left(\begin{array}{cc}
\frac{-J_{2}}{J_{1}\left(J_{1}+J_{2}\right)} & \frac{1}{J_{1}+J_{2}} \\
\frac{1}{J_{1}+J_{2}} & \frac{-J_{1}}{J_{1}\left(J_{1}+J_{2}\right)}
\end{array}\right)\left(\begin{array}{l}
d_{1}(t) \\
d_{2}(t)
\end{array}\right) \\
& =\mathbf{A}\left(\begin{array}{l}
e_{1}(t) \\
e_{2}(t)
\end{array}\right)+\mathbf{B}\left(\begin{array}{c}
r(t) \\
r_{1}(t) \\
r_{2}(t) \\
r_{3}(t)
\end{array}\right)+\overline{\mathbf{B}}\left(\begin{array}{l}
d_{1}(t) \\
d_{2}(t)
\end{array}\right) \\
& \mathbf{A}=\left(\begin{array}{cc}
\frac{k_{11}-k_{31}}{J_{1}} & \frac{k_{12}-k_{32}}{J_{1}} \\
\frac{k_{21}+k_{31}}{J_{2}} & \frac{k_{22}+k_{32}}{J_{2}}
\end{array}\right), \quad \mathbf{B}=\left(\begin{array}{cccc}
\frac{-1}{J_{1}+J_{2}} & \frac{1}{J_{1}} & 0 & \frac{-1}{J_{1}} \\
\frac{-1}{J_{1}+J_{2}} & 0 & \frac{1}{J_{2}} & \frac{1}{J_{2}}
\end{array}\right), \quad \overline{\mathbf{B}}=\left(\begin{array}{cc}
\frac{-J_{2}}{J_{1}\left(J_{1}+J_{2}\right)} & \frac{1}{J_{1}+J_{2}} \\
\frac{1}{J_{1}+J_{2}} & \frac{-J_{1}}{J_{1}\left(J_{1}+J_{2}\right)}
\end{array}\right) .
\end{aligned}
$$

Box I

$u_{1}(t)$ is helpful in the improvement of the engine fuel consumption rate, while less use of $u_{3}(t)$ is helpful in reducing torque interruption and attaining less frictional loss of the clutch. This paper holds the central viewpoint that reduced torque interruption and less frictional loss of the clutch are more important than the optimal fuel consumption rate of the engine during mode shift. Therefore, at the beginning phase of mode shift, it is reasonable to avoid using $u_{3}(t)$ and to select the combination of $\left(u_{1}(t), u_{2}(t)\right)$ as much as possible until the angular speed difference of the driving-driven shafts of the clutch can be regulated to a specific value, $\Delta \varpi_{c}$; then, $u_{3}(t)$ is applied quickly and the combination of $\left(u_{1}(t), u_{2}(t), u_{3}(t)\right)$ is introduced to engage the driving-driven shafts of the clutch. In a word, the suitable combination selection of the control variables based on $\Delta \varpi_{c}$ can be expressed as:

1) When $\left(\omega_{1}(t)-\omega_{2}(t)\right)>\Delta \varpi_{c}$,

Step 1: $\left(u_{1}(t), u_{2}(t)\right)$ is selected,

2) When $\left(\omega_{1}(t)-\omega_{2}(t)\right) \leq \Delta \varpi_{c}$,

Step 2: $\left(u_{1}(t), u_{2}(t), u_{3}(t)\right)$ is selected.

\subsection{Design of the feedforward controller}

Since the control goal is to achieve $\mathbf{e}(t)=0$, the primary purpose of the feedforward controller is to guarantee the error convergence as soon as possible. Therefore, the external reference matrix, $\mathbf{B}(r(t)$, $\left.r_{1}(t), r_{2}(t), r_{3}(t)\right)$, and the external disturbance matrix, $\overline{\mathbf{B}}\left(d_{1}(t), d_{2}(t)\right)$, should tend to zero, and the constraint equations can be given as:

$$
\begin{aligned}
\frac{-r(t)}{J_{1}+J_{2}} & +\frac{r_{1}(t)}{J_{1}}-\frac{r_{3}(t)}{J_{1}}-\frac{J_{2}}{J_{1}\left(J_{1}+J_{2}\right)} d_{1}(t) \\
& +\frac{1}{J_{1}+J_{2}} d_{2}(t)=0, \\
\frac{-r(t)}{J_{1}+J_{2}} & +\frac{r_{2}(t)}{J_{2}}+\frac{r_{3}(t)}{J_{2}}+\frac{1}{J_{1}+J_{2}} d_{1}(t) \\
& -\frac{J_{1}}{J_{2}\left(J_{1}+J_{2}\right)} d_{2}(t)=0 .
\end{aligned}
$$

Based on Eqs. (29) and (30), the constraint equations among $r(t), r_{1}(t), r_{2}(t)$, and $r_{3}(t)$ can be achieved as:

$$
\begin{aligned}
r_{1}(t)+ & r_{2}(t)=r(t), \\
r_{3}(t)= & \frac{-J_{1}}{J_{1}+J_{2}} r(t)+r_{1}(t)-\frac{J_{2}}{J_{1}+J_{2}} d_{1}(t) \\
& +\frac{J_{1}}{J_{1}+J_{2}} d_{2}(t) .
\end{aligned}
$$

\subsection{Design of the feedback controller}

To facilitate the design of six parameters for the feedback controller, other expressions of error equations can 
be derived by Eqs. (19), (20), and (22) as:

$$
\begin{aligned}
e_{1}(t)= & \frac{u_{1}(t)-u_{3}(t)}{J_{1}}-\frac{u_{m}(t)-d_{2}(t)}{J_{1}+J_{2}} \\
& -\frac{2 J_{1}+J_{2}}{J_{1}\left(J_{1}+J_{2}\right)} d_{1}(t), \\
e_{2}(t)= & \frac{u_{2}(t)+u_{3}(t)}{J_{1}}-\frac{u_{m}(t)-d_{1}(t)}{J_{1}+J_{2}} \\
& -\frac{2 J_{2}+J_{1}}{J_{2}\left(J_{1}+J_{2}\right)} d_{2}(t) .
\end{aligned}
$$

Obviously, it is found that $e_{1}(t)$ is independent of $u_{2}(t)$, and $e_{2}(t)$ is irrelevant to $u_{1}(t)$. Therefore, due to the proposed feedback-feedforward control algorithm (23), it is reasonable that parameters $k_{12}$ and $k_{21}$ are supposed to be zero; also, the stability conditions (26) and (27) can be simplified as:

$$
\begin{aligned}
& k_{11}+k_{22}-k_{31}+k_{32}<0 \\
& k_{31} k_{22}-k_{11}\left(k_{22}+k_{32}\right)<0 .
\end{aligned}
$$

In addition, regarding the combination selection of the control variables (Eq. (28)), $u_{3}(t)$ can be assumed as an external disturbance to $x_{2}(t)$ because the clutch friction torque changes from zero to nonzero during mode shift. From Eqs. (19) and (23), the dynamic equation of state variable $x_{2}(t)$ can be expressed as:

$$
J_{2} \dot{x}_{2}(t)=r_{2}(t)+k_{21} e_{1}(t)+k_{22} e_{2}(t)+u_{3}(t)-d_{2}(t) \text {. }
$$

To reduce the disturbance impact of $u_{3}(t)$ on state variable $x_{2}(t), u_{3}(t)$ should supposedly counteract the parts of $k_{21} e_{1}(t)+k_{22} e_{2}(t)$, and the reference variable $r_{2}(t)$ can directly determine the trajectory of $x_{2}(t)$. Therefore, since $k_{21}$ is set to zero, parameter $k_{22}$ can be designed to consist of two parts: an offset value, $k_{22} e$, and a reference value, $k_{22} \_$:

$$
\begin{aligned}
& k_{22}(t)=k_{22 \_e}+k_{22_{-} r}(t), \\
& k_{22 \_r}(t)=-\frac{u_{3}(t)}{e_{2}(t)} .
\end{aligned}
$$

The above-described method to select the parameter $k_{22}$ shows a great potential to explore the design space of the feedback controller. Note that since $k_{22}(t)$ becomes a time-varying parameter, the stability conditions (35) and (36) are not applicable to the control system. However, the design criterion of the linear time-varying system [29] can provide a suitable selection method of $k_{22} e$, and the detailed derivation is not of interest in this section.

\subsection{Discussion on continuity of $u_{3}(t)$}

The clutch friction torque during the slipping-locked process may become discontinuous and change sharply to cause intensive vehicle jerk. Based on the control strategy proposed in this paper, the friction-induced discontinuity of the clutch torque during mode shift can be effectively avoided, if the clutch friction torque $u_{3}(t)$ during the locked process equals the value of the slipping process. This section will discuss the torque continuity of the clutch.

Through combining the MRC algorithm (23) proposed in this paper with Eq. (9), the clutch friction torque during the locked process is denoted by $u_{3}^{+}(t)$ and can be expressed as:

$$
\begin{aligned}
u_{3}^{+}(t)= & \frac{J_{2}}{J_{1}+J_{2}} u_{1}(t)-\frac{J_{1}}{J_{1}+J_{2}} u_{2}(t) \\
& -\frac{J_{2}}{J_{1}+J_{2}} d_{1}(t)+\frac{J_{1}}{J_{1}+J_{2}} d_{2}(t) \\
= & \frac{J_{2}}{J_{1}+J_{2}}\left(r_{1}(t)+k_{11} e_{1}(t)\right) \\
& -\frac{J_{1}}{J_{1}+J_{2}}\left(r_{2}(t)+k_{22} e_{2}(t)\right) \\
& -\frac{J_{2}}{J_{1}+J_{2}} d_{1}(t)+\frac{J_{1}}{J_{1}+J_{2}} d_{2}(t) \\
= & \frac{J_{2}}{J_{1}+J_{2}} r_{1}(t)-\frac{J_{1}}{J_{1}+J_{2}} r_{2}(t) \\
& -\frac{J_{2}}{J_{1}+J_{2}} d_{1}(t)+\frac{J_{1}}{J_{1}+J_{2}} d_{2}(t) \\
& +\frac{J_{2}}{J_{1}+J_{2}} k_{11} e_{1}(t)-\frac{J_{1}}{J_{1}+J_{2}} k_{22} e_{2}(t) .
\end{aligned}
$$

On the other hand, the clutch friction torque during the slipping process is denoted by $u_{3}^{-}(t)$ and can be expressed as:

$$
u_{3}^{-}(t)=r_{3}(t)+k_{31} e_{1}(t)+k_{32} e_{2}(t) .
$$

Substitution of Eqs. (31) and (32) into Eq. (41) can derive:

$$
\begin{aligned}
u_{3}^{-}(t)= & r_{3}(t)+k_{31} e_{1}(t)+k_{32} e_{2}(t) \\
= & \frac{-J_{1}}{J_{1}+J_{2}} r(t)+r_{1}(t)-\frac{J_{2}}{J_{1}+J_{2}} d_{1}(t) \\
& +\frac{J_{1}}{J_{1}+J_{2}} d_{2}(t)+k_{31} e_{1}(t)+k_{32} e_{2}(t) \\
= & \frac{-J_{1}}{J_{1}+J_{2}}\left(r_{1}(t)+r_{2}(t)\right)+r_{1}(t)-\frac{J_{2}}{J_{1}+J_{2}} d_{1}(t)
\end{aligned}
$$




$$
\begin{aligned}
& +\frac{J_{1}}{J_{1}+J_{2}} d_{2}(t)+k_{31} e_{1}(t)+k_{32} e_{2}(t) \\
= & \frac{J_{2}}{J_{1}+J_{2}} r_{1}(t)-\frac{J_{1}}{J_{1}+J_{2}} r_{2}(t)-\frac{J_{2}}{J_{1}+J_{2}} d_{1}(t) \\
& +\frac{J_{1}}{J_{1}+J_{2}} d_{2}(t)+k_{31} e_{1}(t)+k_{32} e_{2}(t) .
\end{aligned}
$$

Because the control goal is to achieve $J:=\left\{\mathbf{e}(t)|| e_{1} \mid+\right.$ $\left.\left|e_{2}\right|=0\right\}$, when the expected control goal is achieved at the end of the clutch-slipping process, the error equations would be equal to zero with $e_{1}(t)=0$ and $e_{2}(t)=0$, and then Eq. (40) can be expressed as:

$$
\begin{aligned}
u_{3}^{+}(t)= & \frac{J_{2}}{J_{1}+J_{2}} r_{1}(t)-\frac{J_{1}}{J_{1}+J_{2}} r_{2}(t) \\
& -\frac{J_{2}}{J_{1}+J_{2}} d_{2}(t)+\frac{J_{1}}{J_{1}+J_{2}} d_{2}(t) \\
& +\frac{J_{2}}{J_{1}+J_{2}} k_{11} e_{1}(t)-\frac{J_{1}}{J_{1}+J_{2}} k_{22} e_{2}(t) \\
= & \frac{J_{2}}{J_{1}+J_{2}} r_{1}(t)-\frac{J_{1}}{J_{1}+J_{2}} r_{2}(t) \\
& -\frac{J_{2}}{J_{1}+J_{2}} d_{2}(t)+\frac{J_{1}}{J_{1}+J_{2}} d_{2}(t) .
\end{aligned}
$$

In addition, the control goal, $\mathbf{e}(t)=0$, means that the vehicle enters the clutch-locked process, and thus Eq. (42) can be expressed as:

$$
\begin{aligned}
u_{3}^{-}(t)= & r_{3}(t)+k_{31} e_{1}(t)+k_{32} e_{2}(t) \\
= & \frac{J_{2}}{J_{1}+J_{2}} r_{1}(t)-\frac{J_{1}}{J_{1}+J_{2}} r_{2}(t)-\frac{J_{2}}{J_{1}+J_{2}} d_{1}(t) \\
& +\frac{J_{1}}{J_{1}+J_{2}} d_{2}(t)+k_{31} e_{1}(t)+k_{32} e_{2}(t) \\
= & \frac{J_{2}}{J_{1}+J_{2}} r_{1}(t)-\frac{J_{1}}{J_{1}+J_{2}} r_{2}(t) \\
& -\frac{J_{2}}{J_{1}+J_{2}} d_{1}(t)+\frac{J_{1}}{J_{1}+J_{2}} d_{2}(t) .
\end{aligned}
$$

According to Eqs. (43) and (44), it is found that the continuity of $u_{3}(t)$ during the slipping-locked transition process can be guaranteed by $u_{3}^{+}(t)=u_{3}^{-}(t)$, which avoids the torque interruption under the control strategy proposed in this paper.

\section{Results and discussion}

\subsection{Simulation results and discussion}

Usually, to complete mode shift under the acceleration condition, many hybrid electric vehicles always apply the method by pressing the accelerator pedal gradually and, then, simultaneously releasing the clutch pedal [30], which is called (Conv.) the conventional control method in this paper. Therefore, to evaluate the performance of the proposed MRC during mode shift, the simulation results of the dual-mode powersplit HEV from EVT 1 to EVT 2 are given by comparison with those of the conventional control method, as shown in Figure 5. The vehicle parameters of the dualmode power-split HEV are listed in Table 2, and the control parameters of the MRC are listed in Table 3. The conventional control method can be expressed as:

$$
u_{1}(t)=T_{1}(t), \quad u_{2}(t)=T_{m}(t), \quad u_{3}(t)=g \cdot t,
$$

in which $g=450$ is chosen for the simulation and it is seen that the clutch friction torque increases at a constant rate during mode shift.

Table 2. Simulation parameters.

\begin{tabular}{lc}
\hline \multicolumn{1}{c}{ Parameter } & Value \\
\hline Vehicle parameters: & $25000 \mathrm{~kg}$ \\
Vehicle mass & $0.318 \mathrm{~m}$ \\
Tire radius & 4.42 \\
Final gear ratio 1 & 4.0 \\
Final gear ratio 2 & 0.025 \\
Rolling resistance coefficient & $1.4 \mathrm{~kg} / \mathrm{m}^{3}$ \\
Air density & 0.6 \\
Air drag coefficient & $4.8 \mathrm{~m}{ }^{2}$ \\
Vehicle front area & \\
Notation parameters: & \\
$K_{1}, K_{2}, K_{3}$ & $2.13,2.13,2.33$ \\
$T_{f}$ & $50 \mathrm{Nm}$ \\
$J_{1}, J_{2}$ & $2.28 \mathrm{~kg} / \mathrm{m}^{2}, 14.24 \mathrm{~kg} / \mathrm{m}^{2}$ \\
$Z$ & 8 \\
$R_{o}, R_{i}$ & $0.334 \mathrm{~m}, 0.286 \mathrm{~m}$ \\
$A_{p}$ & $0.029 \mathrm{~m}{ }^{2}$ \\
$\mu_{d}, \mu_{s}$ & $0.06,0.1$ \\
$\Delta \varpi_{c}$ & $40 \mathrm{rad} / \mathrm{s}$ \\
\hline
\end{tabular}

Table 3. MRC parameters.

\begin{tabular}{ccc}
\hline Parameter & Step 1 & Step 2 \\
\hline$r_{1}(t)$ & 120 & 120 \\
$r_{2}(t)$ & Calculated & Calculated \\
$r_{3}(t)$ & 0 & Calculated \\
$k_{11}, k_{12}$ & $-2.5,0$ & $-2.5,0$ \\
$k_{21}, k_{22 \_}$ & $0,-40$ & $0,-40$ \\
$k_{31}, k_{32}$ & 0,0 & $-0.3,-0.3$ \\
\hline
\end{tabular}


The initial conditions to begin the mode shift for the dual-mode power-split $\mathrm{HEV}$ is that the angular speed of shaft 1 is $160 \mathrm{rad} / \mathrm{s}$ and that of shaft 2 is $400 \mathrm{rad} / \mathrm{s}$, and the shift duration of Conv. is about $3 \mathrm{~s}$ shorter than that of MRC (see Figure $7(\mathrm{e})$ ). However, Figure $7(d)$ shows that the clutch-slipping duration of Conv. is $1.346 \mathrm{~s}$ (from $12 \mathrm{~s}$ to $13.346 \mathrm{~s}$ ), while that of $\mathrm{MRC}$ decreases to $1.064 \mathrm{~s}$ (from $15.142 \mathrm{~s}$ to $16.206 \mathrm{~s}$ ), i.e. $0.272 \mathrm{~s}$ shorter than that of Conv. The mode shift of the dual-mode power-split HEV can produce the clutch friction torque; therefore, the real shift time of MRC should start at $15.142 \mathrm{~s}$ and end at $16.206 \mathrm{~s}$, that is, the MRC can effectively reduce the shift time of the dual-mode power-split HEV by $1.064 \mathrm{~s}$. It is clear that fast friction torque of the clutch can quickly engage the driving-driven shafts of the clutch; this in turn explains why the MRC has a longer shift duration as no friction torque exists before the clutch-slipping process. On the other hand, fast shift duration does not mean short clutch-slipping process for the dual-mode powersplit HEV during mode shift. As a whole, Figure $7(\mathrm{~d})$ and (e) demonstrate that the MRC can simultaneously decrease the shift time and the friction torque.

Vehicle jerk and frictional loss of the clutch can negatively affect the shift quality of the dual-mode power-split HEV during mode shift. The calculation of the vehicle jerk, $j$, and the frictional loss of the clutch, $E$, can be expressed as:

$$
\begin{aligned}
& j=\frac{d a}{d t}=\frac{d^{2} v}{d t^{2}}, \\
& E=\int_{t_{1}}^{t_{2}} a b s\left(\omega_{1}-\omega_{2}\right) \cdot T_{c} d t,
\end{aligned}
$$

where $a$ denotes the vehicle acceleration and $v$ denotes the vehicle speed.

By the comparison of Figure $7(\mathrm{f})$, it is obviously found that the vehicle acceleration remains steady when the MRC is applied, whereas it falls down by about $1.4 \mathrm{~m} / \mathrm{s}^{2}$ when the Conv. is applied. For the Conv., the profile similarity reflects the interaction influence between the friction torque and the vehicle acceleration (see Figure $7(\mathrm{~d})$ and (f)), which means that the friction torque has a negative impact on the vehicle acceleration. Because no torque compensation is activated (see Figure $7(\mathrm{c})$ ), a big torque interruption is generated by the clutch to cause the falling of the vehicle acceleration. On the other hand, for the MRC, MG2 can increase the torque of $T_{2}$ to compensate for the negative impact on the vehicle acceleration and the friction torque is very smaller; therefore, torque interruption is avoided (see Figure $7(\mathrm{c})$ and $(\mathrm{d})$ ).

As shown in Figure $7(\mathrm{~g})$, the vehicle jerk from the Conv. is up to about $37 \mathrm{~m} / \mathrm{s}^{3}$ because the friction torque changes suddenly at the end of the clutch- slipping process. At this point, the vehicle jerk from the MRC is less than $1 \mathrm{~m} / \mathrm{s}^{3}$, even in the slippinglocked transition process. This is because for the $\mathrm{MRC}$, the reference model can coordinately control the friction torque during the slipping process, and the torque continuity of the clutch is also guaranteed by Eqs. (43) and (44). Therefore, the MRC can avoid the abrupt change of the friction torque, thus reducing the intensive vehicle jerk.

As shown in Figure $7(\mathrm{~h})$, the clutch frictional loss of the MRC is about $407 \mathrm{~J}$, while that of the Conv. is up to about $48720 \mathrm{~J}$. The frictional loss is generated during the slipping process, which is mainly determined by three factors based on Eq. (47): the slipping duration, the angular speed difference, and the friction torque. Based on Figure $7(\mathrm{~d})$ and (e), all the factors produced by the MRC are less than those produced by the Conv. Therefore, the clutch frictional loss in MRC is significantly reduced in comparison with that in the Conv.

\subsection{Sensitivity analysis}

To further understand the design implications of the MRC strategy, it is necessary to consider the following key factors that might impact on the mode shift characteristics: the three feedforward parameters $r_{1}(t), r_{2}(t)$, and $r_{3}(t)$; the shift trigger $\Delta \varpi_{c}$; and the estimation errors of the external disturbances $T_{f}(t)$ and $T_{l}(t)$. These key factors are analyzed as follows:

1. Analysis of feedforward parameters $r_{1}(t), r_{2}(t)$, and $r_{3}(t)$. Based on the strategy presented in Section 4.4 , it is seen that the selection principles for the feedforward parameters $r_{1}(t), r_{2}(t), r_{3}(t)$ allow for one degree of freedom for the three feedforward input variables. In other words, if any one of the three feedforward parameters is defined, then the other two can be determined uniquely. Therefore, which variable should be predefined and how to determine its value are relevant questions for real applications. The physical significance of $r_{1}(t)$ and $r_{2}(t)$ is the equivalent torques, $T_{1}(t)$ and $T_{2}(t)$, as $\mathbf{e}(t)$ converges on zero, which involves the engine torque, $T_{e}(t)$, and the MG torques, $T_{\mathrm{MG} 1}(t)$ and $T_{\mathrm{MG} 2}(t)$. Therefore, the values of $r_{1}(t)$ and $r_{2}(t)$ can be predefined based on the energy management strategy that involves the management of the torque distribution.

The example selected in this section is given as: The values of $r_{1}(t)$ are set to 110,120 , and 130; the other parameters are the same as those defined in Table 3 and the desired traction torque is also the same as that in Figure 7(a). The simulation results of the sensitivity analysis of $r_{1}(t)$ are shown in Figure 8. No acceleration loss is found in Figure 8(d) and no sudden jerk occurs in Figure $8(\mathrm{e})$. Figure $8(\mathrm{f})$ shows that $r_{1}(t)=130$ 


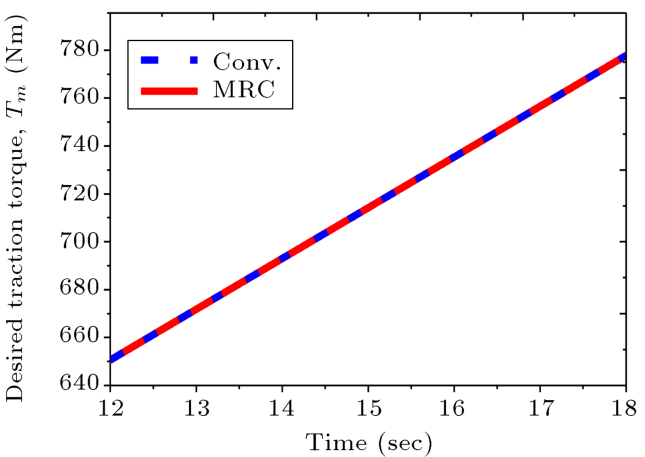

(a)

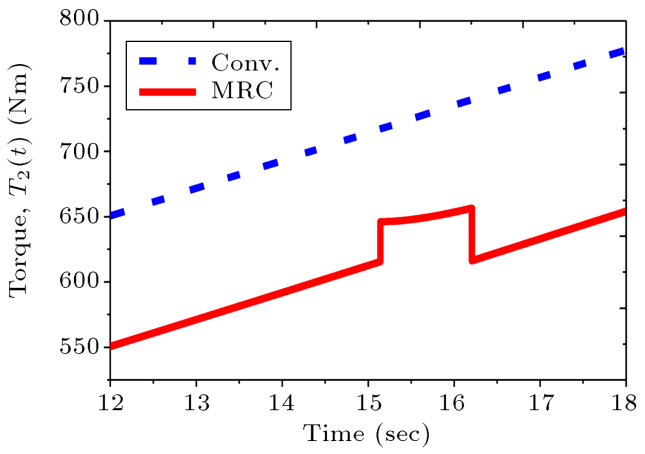

(c)

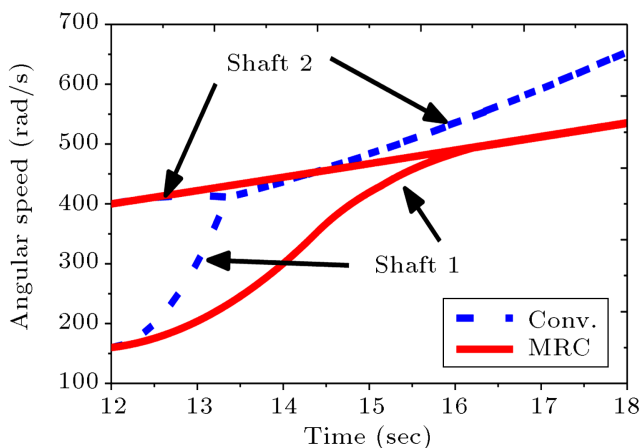

(e)

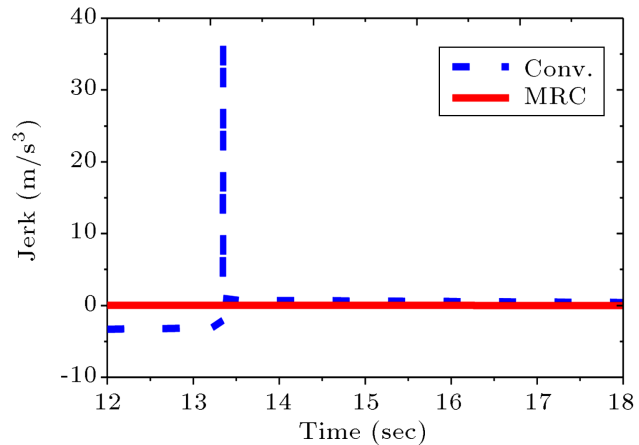

$(\mathrm{g})$

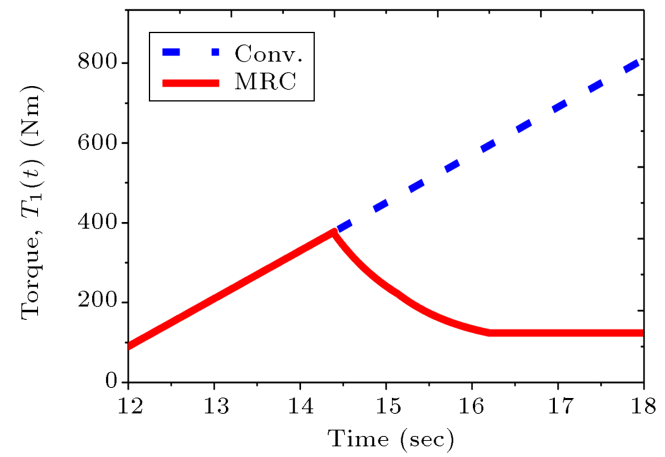

(b)

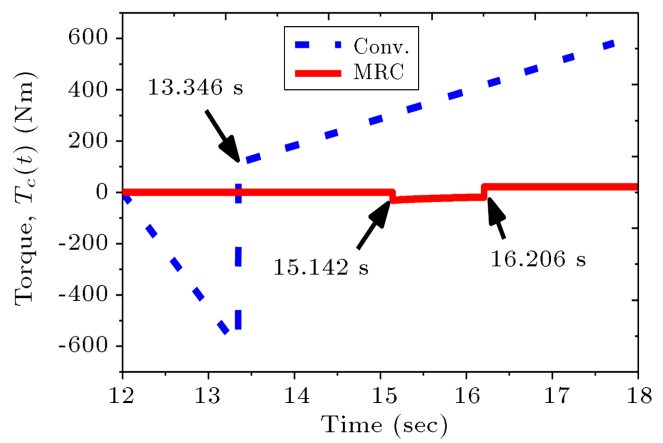

(d)

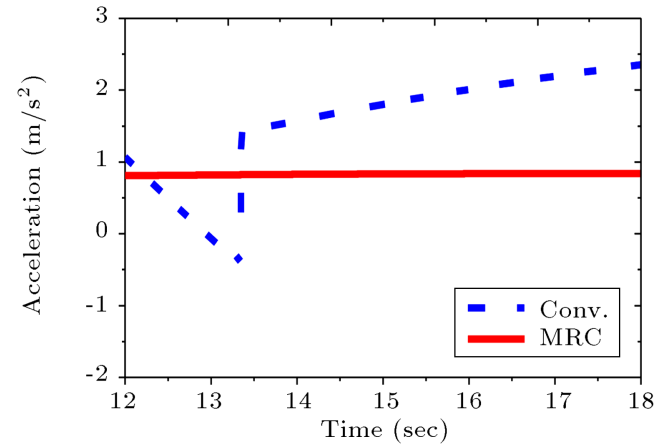

(f)

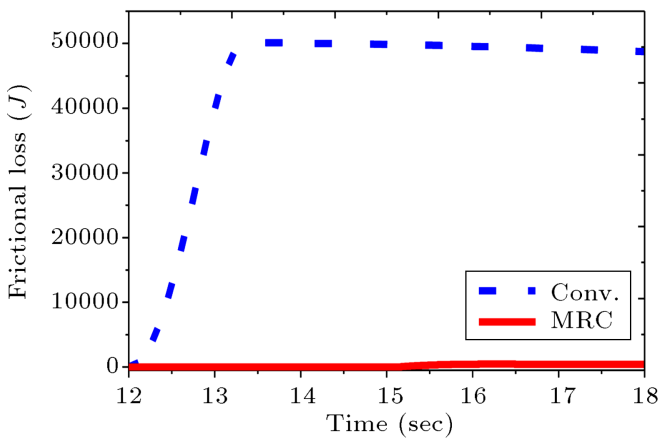

(h)

Figure 7. Comparison between MRC and conventional controller: (a) Desired traction torque, $T_{m}$, (b) equivalent torque, $T_{1}$, (c) equivalent torque, $T_{2}$, (d) clutch torque, $T_{c}$, (e) angular speed, (f) acceleration, (g) vehicle jerk, and (h) frictional loss.

produces about $180 \mathrm{~J}$ more frictional loss than $r_{1}(t)=110$ does; however, the frictional losses are insignificant. Therefore, based on the proposed $\mathrm{MRC}$ in this paper, the different values of $r_{1}(t)$ can maintain good performance of the power-split
HEV during mode shift. The simulation results of different values of $r_{2}(t)$ are similar to those of $r_{1}(t)$. In conclusion, the mode shift characteristics of the proposed MRC in this paper are not sensitive to different torque distribution strategies, and it is a 


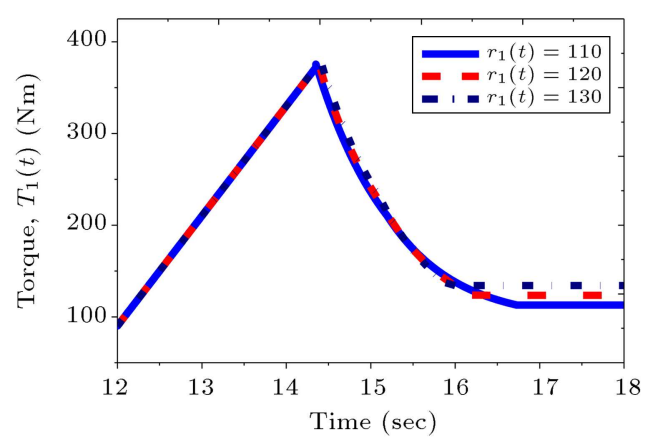

(a)

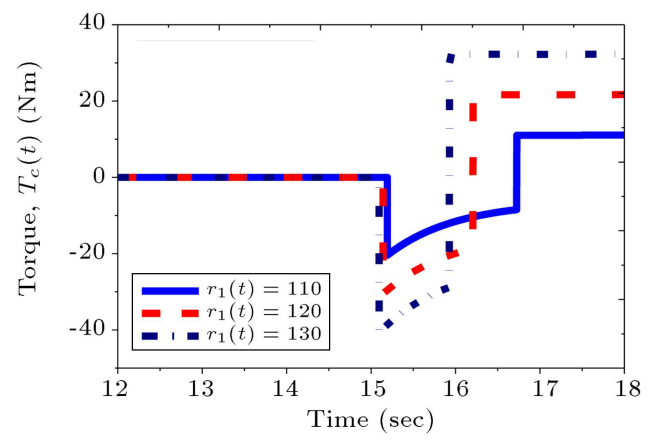

(c)

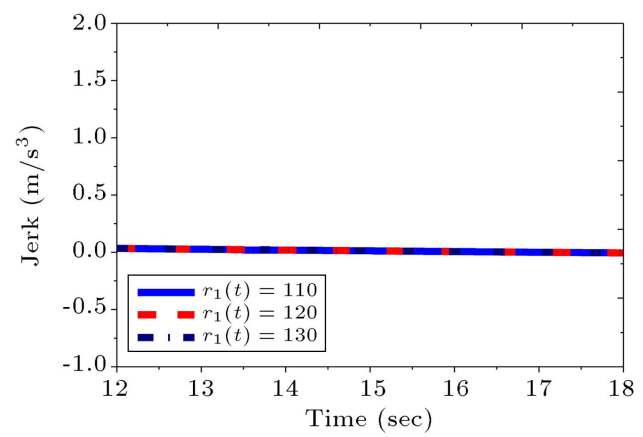

(e)

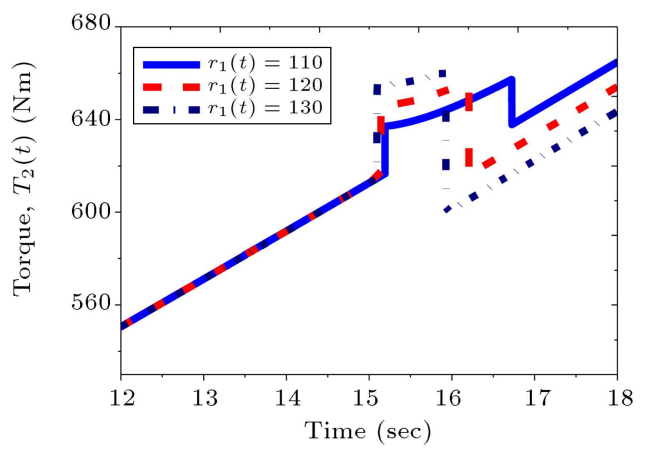

(b)

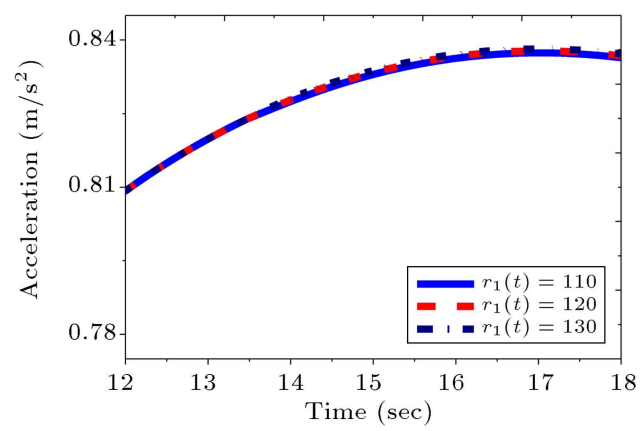

(d)

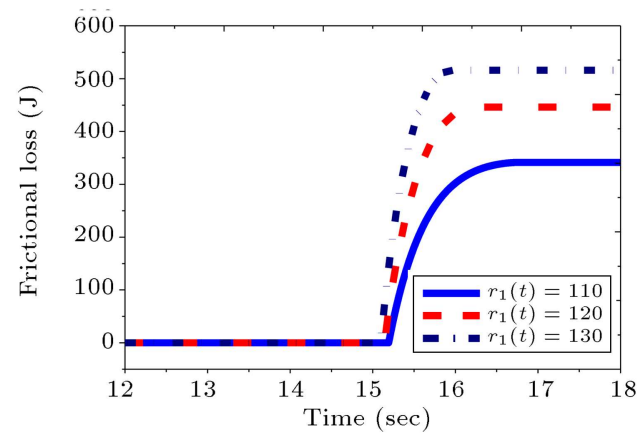

(f)

Figure 8. Sensitivity analysis of $r_{1}(t)$ : (a) Equivalent torque, $T_{1}$, (b) equivalent torque, $T_{2}$, (c) clutch torque, $T_{c}$, (d) acceleration, (e) vehicle jerk, and (f) frictional loss.

significant feature of energy management for the power-split HEV;

2. Analysis of shift trigger $\Delta \varpi_{c}$. The example selected in this section is given as: the values of $\Delta \varpi_{c}$ are set to 40, 70, and 100; the other parameters are the same as those defined in Table 3 and the desired traction torque is also the same as that in Figure $7(\mathrm{a})$. It is seen that larger values of $\Delta \varpi_{c}$ can produce the clutch torque at an earlier stage in Figure 9(c) and (b) to compensate for $T_{2}(t)$. No acceleration loss and no sudden jerk occur for different values of $\Delta \varpi_{c}$ during mode shift, as shown in Figure $9(\mathrm{~d})$ and (e). However, $\Delta \varpi_{c}=100$ produces about $1500 \mathrm{~J}$ more frictional loss than $\Delta \varpi_{c}=40$ does, which implies that the frictional loss is greatly affected by $\Delta \varpi_{c}$. Therefore, small value of $\Delta \varpi_{c}$ can reduce the use of the clutch torque and produce fewer frictional loss. In addition, small value of $\Delta \varpi_{c}$ can decrease the shift time for the power-split HEV during mode shift (see Figure 9(c));

3. Analysis of external disturbances, $T_{f}(t)$ and $T_{l}(t)$. Usually, it is difficult to accurately measure the external disturbances, $T_{f}(t)$ and $T_{l}(t)$, in real system. Here, Gaussian white noises are used by the noise bandwidths of $30 \mathrm{~Hz}$ for $T_{f}(t)$ and $20 \mathrm{~Hz}$ for $T_{l}(t)$, and the noise variances of 6 for $T_{f}(t)$ and 100 for $T_{l}(t)$. The simulation results are shown in Figure 10.

It is seen that $T_{f}(t)$ with noise cannot produce acceleration loss and sudden jerk for the powersplit HEV during mode shift, while $T_{l}(t)$ with noise introduces obvious vibrations for the vehicle acceleration and jerk. It is because that $T_{f}(t)$ is 


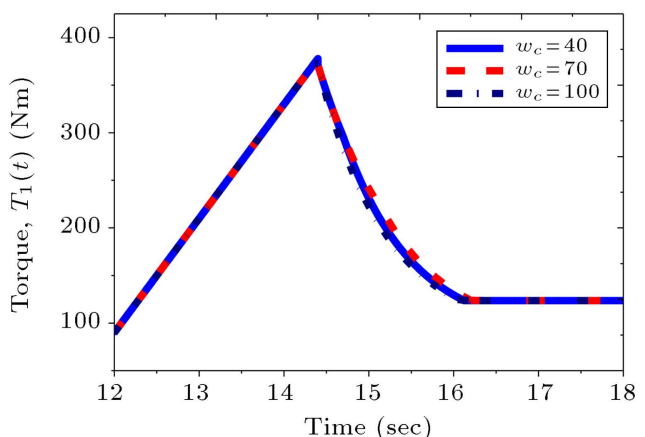

(a)

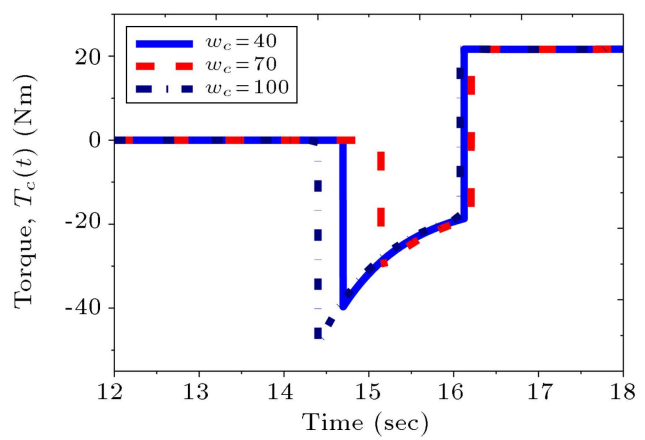

(c)

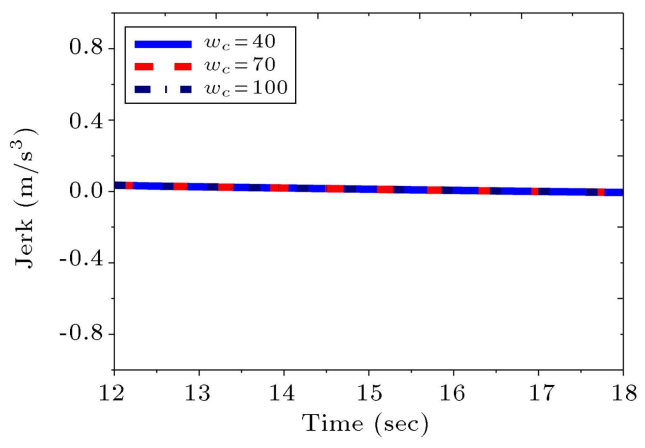

(e)

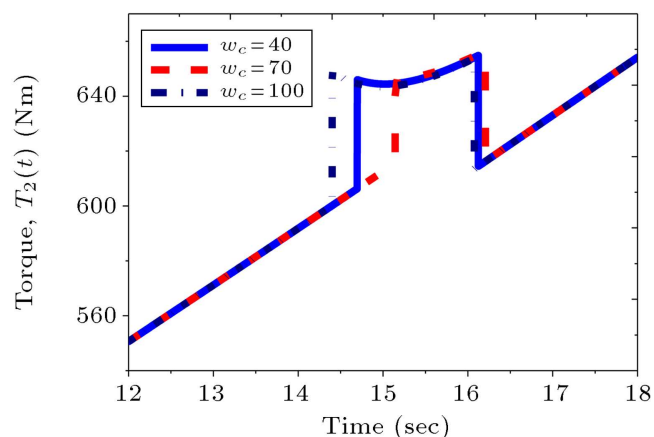

(b)

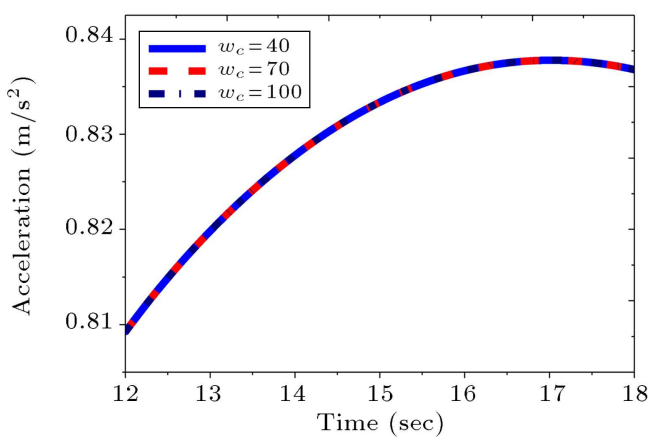

(d)

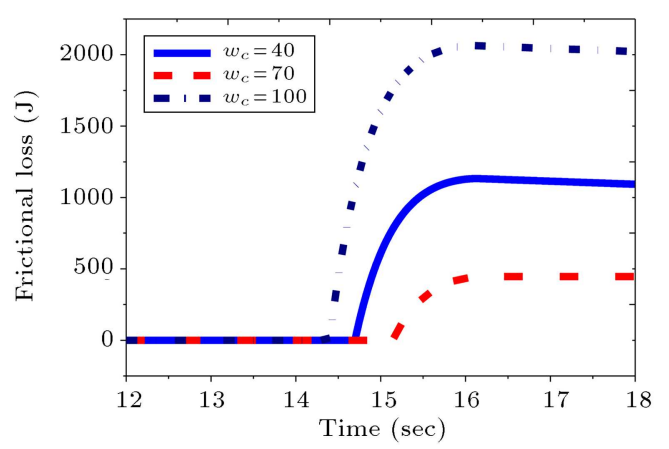

(f)

Figure 9. Sensitivity analysis of $\Delta \varpi_{c}$ : (a) Equivalent torque, $T_{1}$, (b) equivalent torque, $T_{2}$, (c) clutch torque, $T_{c}$, (d) acceleration, (e) vehicle jerk, and (f) frictional loss.

transmitted to the vehicle by the clutch, whereas $T_{l}(t)$ directly acts on the vehicle. Based on the proposed MRC in this paper, $T_{f}(t)$ with noise cannot be passed to the vehicle through the clutch torque, but $T_{l}(t)$ with noise has a direct impact on the mode shift characteristics.

\subsection{Experimental results and discussion}

To further validate the effectiveness of the proposed MRC in this paper, the bench test of the dual-mode power-split HEV is constructed and implemented for test on the mode shift characteristics. Figure 11(a) shows the data acquisition devices that involve using dSPACE and ProtroniC, which can transmit control signals and experimental data between the controllers and the bench test, and Figure 11(b) shows the overall powertrain configuration of the bench test.

The controllers of Conv. and MAC are carried out for the bench test of the dual-mode power-split HEV. Due to inaccuracy of the physical parameters of the model and the discretization and sampling of the controllers, there exists some differences between the experimental results and the simulation results. As shown in Figure 12, it is observed that no vibration other than the vehicle jerk is seen in the simulation results, while considerable vibration exists in the experimental results. That is because the damping and elastic elements of the driveline for reducing jerk are ignored in the simulation model, since this paper emphasizes the effect of the clutch slipping-locked transition on the vehicle jerk; therefore, only the vehicle jerk is reflected in the simulation results. However, the damping and elastic elements do exist in the driveline system of the bench test, and vibration is found in the experimental results.

By comparison with the simulation results, the 


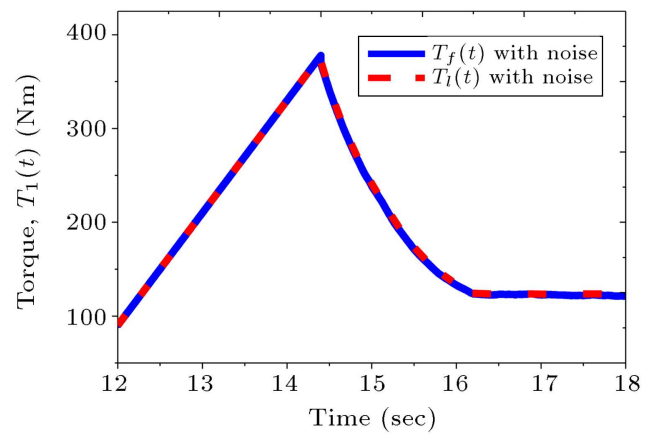

(a)

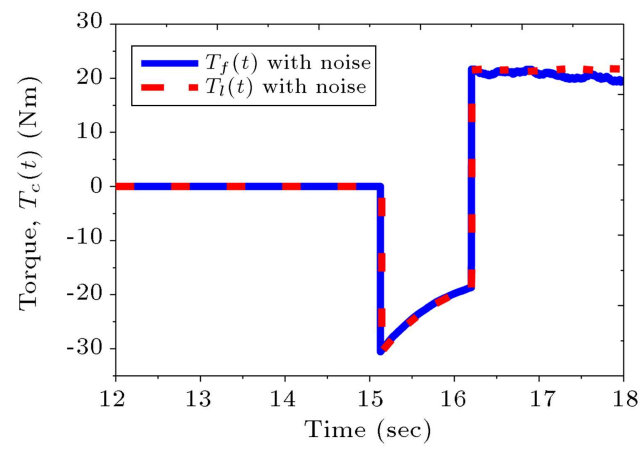

(c)

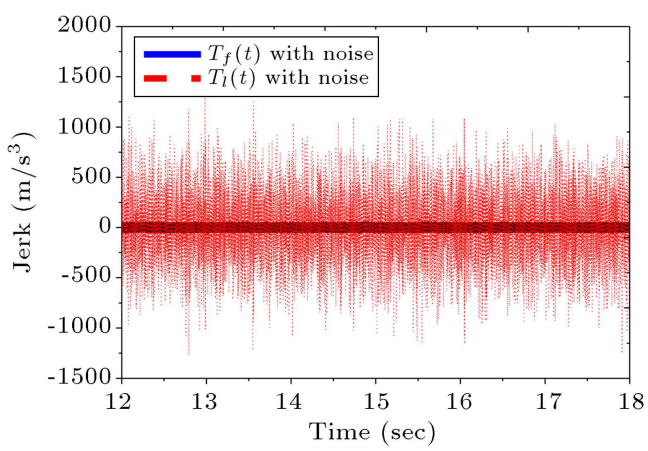

(e)

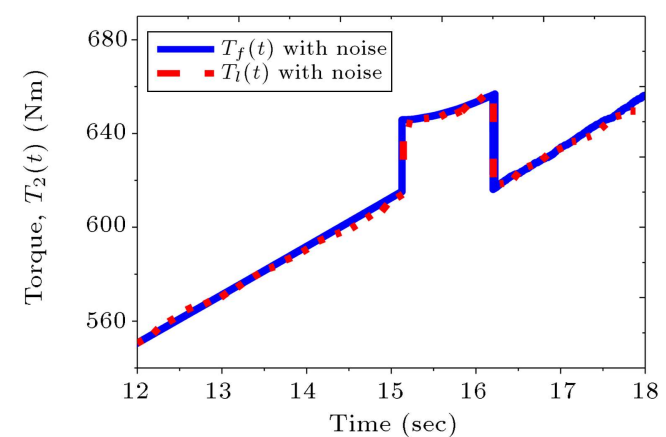

(b)

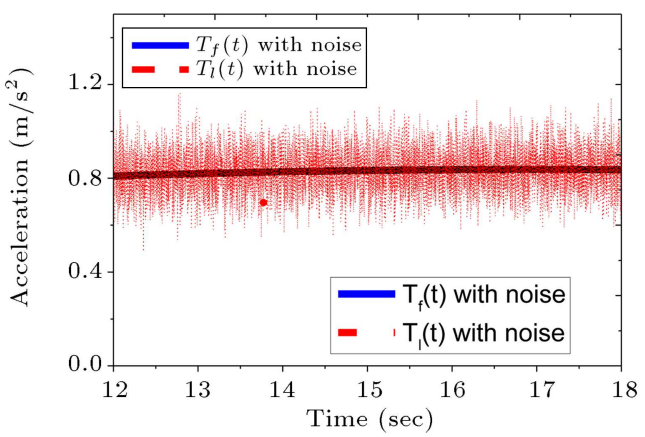

(d)

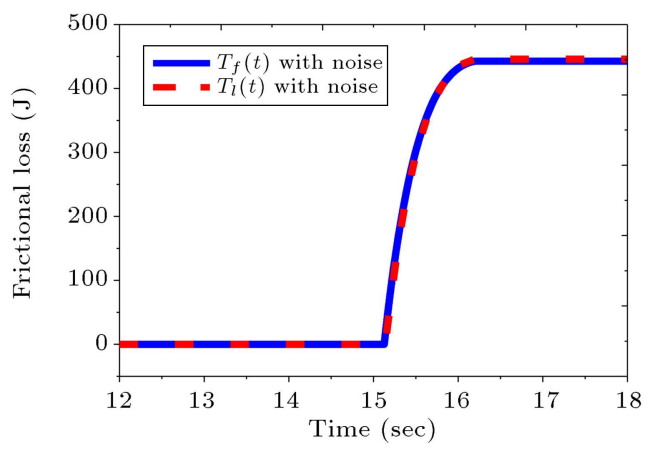

(f)

Figure 10. Sensitivity analysis of $T_{f}(t)$ and $T_{l}(t)$ : (a) Equivalent torque, $T_{1}$, (b) equivalent torque, $T_{2}$, (c) clutch torque, $T_{c},(\mathrm{~d})$ acceleration, (e) vehicle jerk, and (f) frictional loss.

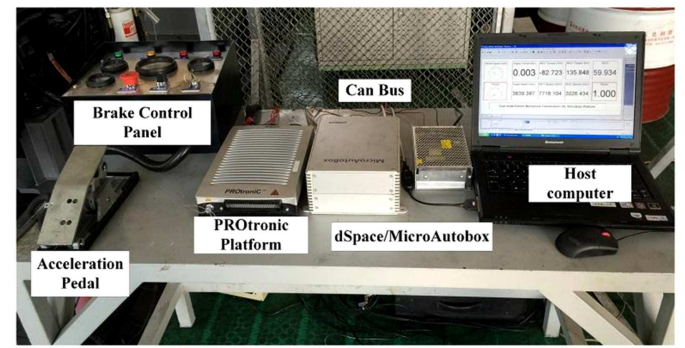

(a)

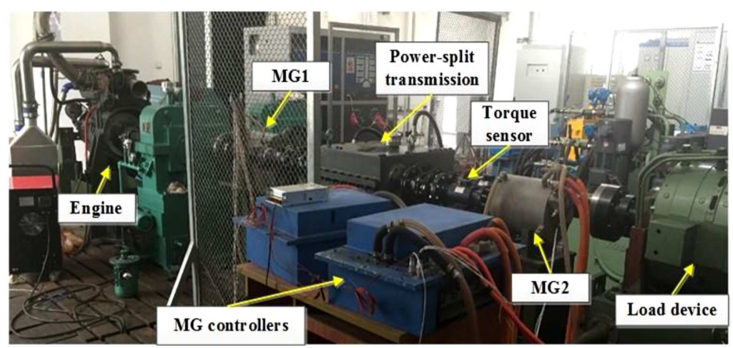

(b)

Figure 11. Bench test for the dual-mode power-split HEV: (a) Data acquisition devices and (b) bench test configuration.

experimental results show the improvement potential of the MRC. For the MRC, the clutch-slipping duration (shift time) is about $0.86 \mathrm{~s}$ and the acceleration fluctuation is about $0.1 \mathrm{~m} / \mathrm{s}^{2}$, while these values are about $1.07 \mathrm{~s}$ and $2.6 \mathrm{~m} / \mathrm{s}^{2}$, respectively, for the Conv. (see Figure 12(e) and (f)). In addition, errors related to discretization and sampling in digital controller as well as limitations in clutch actuation lead to a relatively small impulse input to the driveline and a slight vehicle jerk from the MRC occurs during mode 

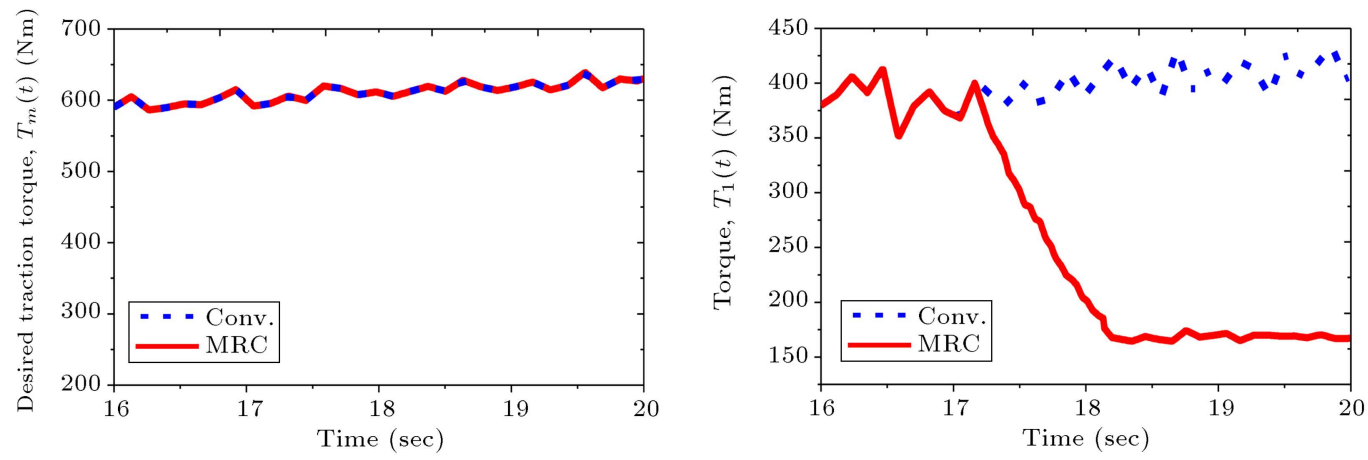

(a)

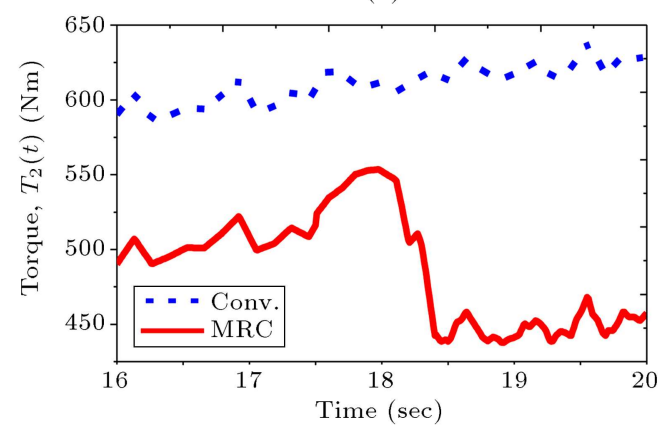

(b)

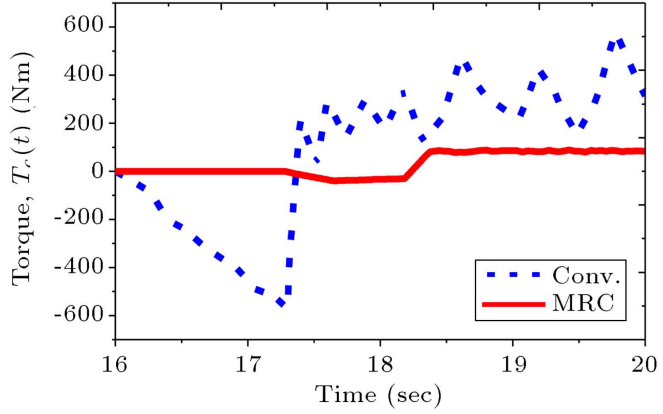

(c)

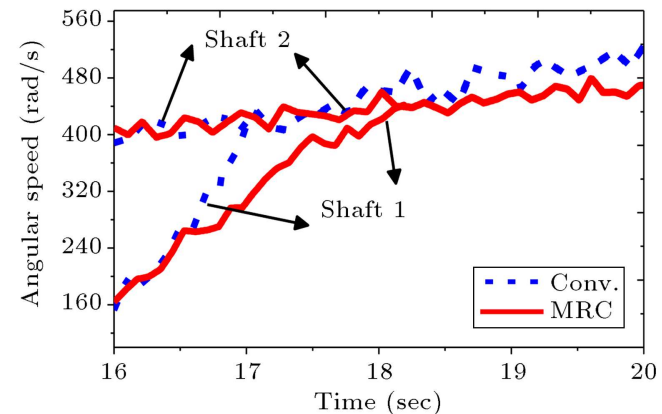

(d)

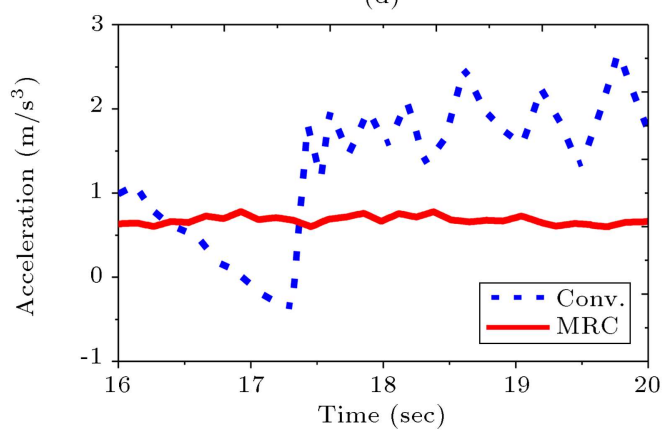

(f)

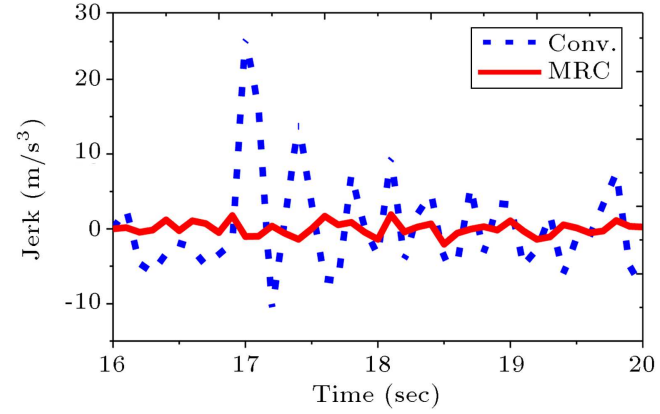

(g)

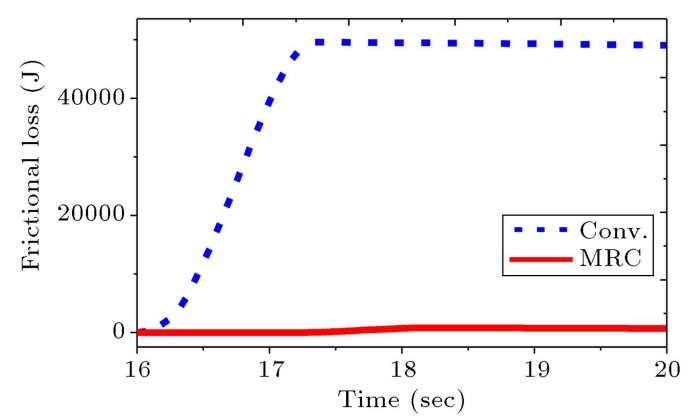

(h)

Figure 12. Comparison between MRC and conventional controller: (a) Desired traction torque, $T_{m}$, (b) equivalent torque, $T_{1}$, (c) equivalent torque, $T_{2}$, (d) clutch torque, $T_{c}$, (e) angular speed, (f) acceleration, (g) vehicle jerk, and (h) frictional loss.

shift; however, it is much less than that by the Conv. (see Figure $12(\mathrm{~g})$ ). The frictional loss of the MRC is about $660 \mathrm{~J}$, while that of the Conv. is about $49500 \mathrm{~J}$ (see Figure 12(h)).

In conclusion, the simulation results and the experimental results demonstrate that the proposed MRC in this paper can simultaneously reduce the vehicle jerk and the clutch frictional loss, thereby improving the shift quality.

\section{Conclusion}

In this paper, a Model Reference Controller (MRC) of mode shift for the dual-mode power-split HEV was 
proposed to achieve smooth mode shift and improved shift quality through reducing the vehicle jerk and the clutch frictional loss. To facilitate the controller design, a control-oriented model capturing the mode shift dynamics of the dual-mode power-split HEV was developed. To simplify the mode shift process, the switched system theory was applied to partition the state space of mode shift into domains that provided a systematic methodology for the controller design. To define the expected control goal, a reference model was introduced to generate the feedback signals, and the error equations were derived between the control plant and the reference model. Moreover, the stability conditions of the controller could be guaranteed by the Lyapunov stability criterion. Because the control system was overactuated with three control variables and two output variables, the systematic parameter selection methods were designed to select a proper combination of the control variables and the MRC parameters. Based on the proposed control strategy and the parameter selection methods, the torque continuity of the clutch could be guaranteed to avoid torque interruption.

To evaluate the performance of the proposed $\mathrm{MRC}$ in this paper, simulation was carried out. The simulation results demonstrated that the frictioninduced discontinuity of the clutch torque during mode shift could be avoided with reduced vehicle jerk and less clutch frictional loss. Moreover, the key factors could affect the performance of the MRC in the powersplit HEV during mode shift, which were studied by the simulations. It was found that the proposed MRC could produce good performance for different values of feedforward parameters, that is, the MRC was compatible with different torque distribution strategies of energy management. The small shift trigger had a significant role in reducing frictional loss and the load disturbance with noise could produce obvious vibration in the vehicle.

In addition, a bench test of the dual-mode powersplit HEV was developed to test the mode shift characteristics. The experimental results approved that the MRC controller could simultaneously achieve reduced vehicle jerk and less clutch frictional loss, when compared with the conventional controller, thereby improving the shift quality. The promising results, particularly the experimental validation, have motivated us to further pursue the idea of MRC and seek its robust and effective implementation in other mode shift control problems with other types of HEV.

\section{Acknowledgements}

The authors are grateful for the financial support from the National Natural Science Foundation of China (No. Grant 51205020).

\section{References}

1. Liu, J. and Peng, H. "Modeling and control of a power-split hybrid vehicle", IEEE Trans. Control. Syst. Technol., 16(6), pp. 1242-1251 (2011).

2. Wang, W., Xiang, C., Liu, H. and Jia, S. "A modelpredictive-control-based power management strategy for a power-split electromechanical transmission", Proc. Inst. Mech. Eng. Part D J. Automob. Eng., 230(14), pp. 1987-2001 (2016).

3. Xiang, C., Huang, K., Ma, Y., Liu, H. and Jia, S. "Analysis of characteristics for mode switch of dual-mode electro-mechanical transmission (EMT)", In 2014 IEEE 80th Veh. Technol. Conf., Vancouver, Canada, pp. 1-6 (2014).

4. Syed, F.U., Kuang, M.L., Czubay, J. and Ying, H. "Derivation and experimental validation of a powersplit hybrid electric vehicle model", IEEE Trans. Veh. Technol., 55(6), pp. 1731-1746 (2006).

5. Kim, J., Kim, T., Min, B., Hwang, S. and Kim, H. "Mode control strategy for a two-mode hybrid electric vehicle using electrically variable transmission (EVT) and fixed-gear mode", IEEE Trans. Veh. Technol., 60(3), pp. 793-803 (2011).

6. Kim, H., Choi, J. and Yi, K. "Development of supervisory control strategy for optimized fuel consumption of the compound hybrid excavator", Proc. Inst. Mech. Eng. Part D J. Automob. Eng., 226(12), pp. 1652-1666 (2012).

7. Yu, H.S., Zhang, J.W. and Zhang, T. "Control strategy design and experimental research on a four-shaft electronic continuously variable transmission hybrid electric vehicle", Proc. Inst. Mech. Eng. Part D J. Automob. Eng., 226(12), pp. 1594-1612 (2012).

8. Yang, C., Song, J., Li, L., Li, S. and Cao, D. "Economical launching and accelerating control strategy for a single-shaft parallel hybrid electric bus", Mech. Syst. Signal. Process., 76-77, pp. 649-664 (2016).

9. Liang, L.I., You, S. and Yang, C. "Multi-objective stochastic MPC-based system control architecture for plug-in hybrid electric buses", IEEE Trans. Industr. Inform., 63(8), pp. 4752-4763 (2016).

10. Kim, N.D., Kim, J.M. and Kim, H.S. "Control strategy for a dual-mode electromechanical, infinitely variable transmission for hybrid electric vehicles", Proc. Inst. Mech. Eng. Part D J. Automob. Eng., 222(9), pp. 1587-1601 (2008).

11. Kang, J., Choi, W. and Kim, H. "Development of a control strategy based on the transmission efficiency with mechanical loss for a dual mode power split-type hybrid electric vehicle", Int. J. Automot. Technol., 13(5), pp. 825-833 (2012).

12. Mashadi, B. and Emadi, S.A. "Dual-mode power-split transmission for hybrid electric vehicles", IEEE Trans. Veh. Technol., 59(7), pp. 3223-3232 (2010).

13. Wu, M.X., Zhang, J.W., Lu, T.L. and Ni, C.S. "Research on optimal control for dry dual-clutch engagement during launch", Proc. Inst. Mech. Eng. Part D J. Automob. Eng., 224(6), pp. 749-763 (2010). 
14. Beck, R., Richert, F., Bollig, A., Abel, D., Saenger, S., Neil, K., Scholt, T., and Noreikat, K.E. "Model predictive control of a parallel hybrid vehicle drivetrain", In Proc. 44th IEEE Conf. Decision Control, Eur. Control Conf., Seville, Spain, pp. 2670-2675 (2005).

15. Minh, V.T., Hashim, F.M. and Awang, M. "Development of a real-time clutch transition strategy for a parallel hybrid electric vehicle", Proc. Inst. Mech. Eng. Part I J. Syst. and Control. Eng., 226(2), pp. 188-203 (2012).

16. Kim, S., Park, J., Hong, J., Lee, M. and Sim, H. "Transient control strategy of hybrid electric vehicle during mode change", SAE Technical Paper (2009).

17. Sanada, K., Gao, B., Kado, N., Takamatsu, H. and Toriya, K. "Design of a robust controller for shift control of an automatic transmission", Proc. Inst. Mech. Eng. Part D J. Automob. Eng., 226(12), pp. 1577-1584 (2012).

18. Gibson, A. and Kolmanovsky, I. "Modeling and analysis of engine torque modulation for shift quality improvement", SAE Technical Paper (2006).

19. Hong, S., Choi, W., Ahn, S., Kim, Y. and Kim, H. "Mode shift control of a dual-mode power-split-type hybrid electric vehicle", Proc. Inst. Mech. Eng. Part D J. Automob. Eng., 228(10), pp. 1217-1231 (2014).

20. Alt, B., Antritter, F., Svaricek, F. and Schultalbers, M. "Multivariable speed synchronisation for a parallel hybrid electric vehicle drivetrain", Vehicle. Syst. Dyn., 51(3), pp. 321-337 (2013).

21. Choi, W., Kang, J., Hong, S. and Kim, H. "Development of a control algorithm to reduce torque variation for the dual-mode HEV during mode shift", In 2011 IEEE Veh. Power. And Propulsion. Conf., Chicago, USA, pp. 1-6 (2011).

22. Hong, S., Choi, W., Ahn, S., Kim, Y. and Kim, H. "Mode shift control of a dual-mode power-split-type hybrid electric vehicle", Proc. Inst. Mech. Eng. Part D J. Automob. Eng., 228(10), pp. 1217-1231 (2014).

23. Song, M., Oh, J., Choi, S., Kim, Y. and Kim, H. "Motor control of a parallel hybrid electric vehicle during mode change without an integrated starter generator", J. Electr. Eng. Technol., 8(4), pp. 930-937 (2013).

24. Kum, D., Peng, H. and Bucknor, N.K. "Control of engine-starts for optimal drivability of parallel hybrid electric vehicles", J. Dyn. Syst. Meas. Control., 135(2), 021020 (2013).

25. Koprubasi, K., Westervelt, E.R. and Rizzoni, G. "Toward the systematic design of controllers for smooth hybrid electric vehicle mode changes", In Proc. Amer. Control. Conf., New York, USA, pp. 2985-2990 (2007).

26. Wang, H., Liu, H. and Yang, J.B. "Dynamic analysis of a two-stage supply chain-a switched system theory approach", Int. J. Adv. Manuf. Technol., 43(1-2), pp. 200-210 (2009).

27. Zhang, H., Shi, Y. and Mehr, A.S. "Stability and stabilization in switched discrete-time systems", Int. J. Adapt. Control. Signal. Process., 26(11), pp. 9911012 (2012).

28. Sun, Z. and Ge, S.S. "Analysis and synthesis of switched linear control systems", Automatica, 41(2), pp. 181-195 (2005).

29. Amato, F., Robust Control of Linear Systems Subject to Uncertain Time-Varying Parameters, Berlin, Germany (2006).

30. Chen, L., Xi, G. and Sun, J. "Torque coordination control during mode transition for a series-parallel hybrid electric vehicle", IEEE Trans. Veh. Technol., 61(7), pp. 2936-2949 (2012).

\section{Biographies}

Changle Xiang was born in Anhui, China, in 1963. He received the $\mathrm{BS}, \mathrm{MS}$, and $\mathrm{PhD}$ degrees in Vehicle Engineering from Beijing Institute of Technology, Beijing, China, in 1984, 1987, and 2001, respectively. $\mathrm{He}$ is currently a Professor with Beijing Institute of Technology, Beijing, China, where he is also the Vice President of Beijing Institute of Technology and the Director of the National Key Laboratory on Vehicular Transmission. He is also currently Chief Designer of Chinese High-Tech Project Group. His research interests include the control of automotive electronics and electric vehicles, particularly research and development of hybrid electric vehicles.

Kun Huang was born in Henan, China, in 1991. He received the BS degree in Vehicle Engineering from Harbin Institute of Technology, Harbin, China, in 2012. $\mathrm{He}$ is currently working toward the $\mathrm{PhD}$ degree in the School of Mechanical Engineering, Beijing Institute of Technology, Beijing, China. Currently, he is also with the National Key Laboratory on Vehicular Transmission, Beijing Institute of Technology. His research interests include modeling and control of hybrid electric vehicles. 